ARTICLE

https://doi.org/10.1038/s41467-019-11403-6

\title{
ARID1A and PI3-kinase pathway mutations in the endometrium drive epithelial transdifferentiation and collective invasion
}

Mike R. Wilson ${ }^{1,6}$, Jake J. Reske ${ }^{1,6}$, Jeanne Holladay ${ }^{1}$, Genna E. Wilber ${ }^{1}$, Mary Rhodes², Julie Koeman², Marie Adams ${ }^{2}$, Ben Johnson ${ }^{3}$, Ren-Wei Su${ }^{1}$, Niraj R. Joshi ${ }^{1}$, Amanda L. Patterson', Hui Shen ${ }^{4}$, Richard E. Leach ${ }^{1,5}$, Jose M. Teixeira (1) 1,5, Asgerally T. Fazleabas ${ }^{1,5}$ \& Ronald L. Chandler ${ }^{1,4,5}$

ARID1A and PI3-Kinase (PI3K) pathway alterations are common in neoplasms originating from the uterine endometrium. Here we show that monoallelic loss of ARID1A in the mouse endometrial epithelium is sufficient for vaginal bleeding when combined with PI3K activation. Sorted mutant epithelial cells display gene expression and promoter chromatin signatures associated with epithelial-to-mesenchymal transition (EMT). We further show that ARID1A is bound to promoters with open chromatin, but ARID1A loss leads to increased promoter chromatin accessibility and the expression of EMT genes. PI3K activation partially rescues the mesenchymal phenotypes driven by ARID1A loss through antagonism of ARID1A target gene expression, resulting in partial EMT and invasion. We propose that ARID1A normally maintains endometrial epithelial cell identity by repressing mesenchymal cell fates, and that coexistent ARID1A and PI3K mutations promote epithelial transdifferentiation and collective invasion. Broadly, our findings support a role for collective epithelial invasion in the spread of abnormal endometrial tissue.

\footnotetext{
${ }^{1}$ Department of Obstetrics, Gynecology, and Reproductive Biology, College of Human Medicine, Michigan State University, Grand Rapids, MI 49503, USA. ${ }^{2}$ Genomics Core Facility, Van Andel Research Institute, Grand Rapids, MI 49503, USA. ${ }^{3}$ Bioinformatics and Biostatistics Core Facility, Van Andel Research Institute, Grand Rapids, MI 49503, USA. ${ }^{4}$ Center for Epigenetics, Van Andel Research Institute, Grand Rapids, MI 49503, USA. ${ }^{5}$ Department of Women's Health, Spectrum Health System, Grand Rapids, MI 49341, USA. ${ }^{6}$ These authors contributed equally: Mike R. Wilson, Jake J. Reske. Correspondence and requests for materials should be addressed to R.L.C. (email: rlc@msu.edu)
} 
T he endometrium is the dynamic inner layer of the uterus, composed of stroma and epithelial cells that undergo monthly proliferation, differentiation, and shedding throughout the menstrual cycle in reproductive age women ${ }^{1}$. Disruption of normal endometrial processes results in a number of pathologies, including endometrial hyperplasia, endometrial cancer $(\mathrm{EC})^{2}$, endometriosis ${ }^{3}$, adenomyosis ${ }^{4}$, and endometriosisassociated ovarian cancer (EAOC) ${ }^{5}$. An estimated 63,230 women will be diagnosed with EC this year ${ }^{6}$, making it the most commonly diagnosed gynecologic malignancy. Furthermore, EC incidence is rising due to the increasing prevalence of obesity ${ }^{2,7}$.

The SWI/SNF chromatin remodeling complex is mutated in $>20 \%$ of all human cancers ${ }^{8,9}$, and the ARID1A (BAF250A) subunit is particularly prone to mutation in gynecologic cancer ${ }^{5,10-13}$. ARID1A mutations are found in $40 \%$ of low-grade $\mathrm{EC}^{12}$, while ARID1A protein expression is lost in 26-29\% of lowgrade and $39 \%$ of high-grade EC ${ }^{13}$. ARID1A loss is observed in focal areas of atypical endometrial hyperplasia ${ }^{14}$, indicating clonal loss. Loss of ARID1A in complex atypical hyperplasia is associated with malignant transformation and concurrent $\mathrm{EC}^{15}$. ARID1A mutations are observed in $11 \%$ of endometriosis and $>30 \%$ of EAOCs $3,5,16,17$. These data support a tumor suppressor role for ARID1A-containing SWI/SNF complexes in neoplasms originating from the endometrium.

Among highly mutated tumor suppressor genes, ARID1A is unique because ARID1A knockout mice are embryonic lethal in the heterozygous state ${ }^{18}$, while other tumor suppressor genes (e.g., TP53) are non-essential for mouse development ${ }^{19}$. ARID1A-null embryos die at embryonic day (E) E6.5 ${ }^{18}$, while DNA-binding defective ARID1A ${ }^{\mathrm{V} 1068 \mathrm{G}}$ mutant embryos die around $\mathrm{E} 10^{20}$. ARID1A mutations are often nonsense and result in a frameshift of the open-reading frame ${ }^{10}$, a characteristic of many tumor suppressors.

Mutations leading to PI3K/AKT pathway upregulation are frequent in $\mathrm{EC}^{21}$, with $84 \%$ of patients displaying mutations in PIK3CA, PIK3R1, or PTEN 22 . PIK3CA mutations commonly cooccur with ARID1A loss in $\mathrm{EC}^{23}$. However, PIK3CA mutations have been observed in normal endometrium ${ }^{17}$. Missense mutations of PIK3CA are common in complex atypical hyperplasia, and PIK3CA mutation has been identified as an early event in endometrial carcinogenesis ${ }^{24}$.

Genetically engineered mouse models (GEMMs) offer the opportunity to study gynecologic pathologies in vivo ${ }^{25-28}$. ARID1A loss in the mouse ovarian surface epithelium drives tumorigenesis when paired with PTEN loss or PIK3CA $\mathrm{C}^{\mathrm{H} 1047 \mathrm{R}}$ mutation $^{29,30}$. In this study, we utilize lactotransferrin-Cre (LtfCre) to target ARID1A mutations and PIK3CA ${ }^{\mathrm{H} 1047 \mathrm{R}}$ directly to the endometrial epithelium. Utilizing the Arid1afl and Arid1 $a^{V 1068 G}$ alleles, we develop an allelic series of loss of function ARID1A mutations in the endometrium, each with increasing severity. We employ genome-wide approaches to profile gene expression and chromatin accessibility of sorted endometrial epithelial cells in vivo and identified chromatin accessibility changes at promoters upon ARID1A loss, which correlate with changes in transcription. Using chromatin immunoprecipitation sequencing (ChIP-seq), we show that ARID1A binding correlates with chromatin accessibility and is associated with gene expression changes upon loss of ARID1A. We utilize human endometrial epithelial cells to elucidate the consequences of ARID1A loss and PIK3CA ${ }^{\mathrm{H} 1047 \mathrm{R}}$ in vitro, and discover a mechanism by which ARID1A and PIK3CA mutations result in a partial EMT phenotype capable of collective invasion into the uterine myometrium. In this context, we characterize the role of ARID1A in epithelial cell identity of the endometrium.

\section{Results}

ARID1A is haploinsufficient in the endometrial epithelium. ARID1A has been hypothesized to function as a haploinsufficient tumor suppressor ${ }^{31}$. To explore this further, we utilized publicly available Uterine Corpus Endometrial Carcinoma (UCEC) mutation and copy-number datasets from The Cancer Genome Atlas (TCGA). Most endometrioid EC patients with ARID1A mutations (either single or multiple hits) show no detectable copy-number alterations at the ARID1A locus, with 33\% of all patients having a single nonsense mutation and normal ploidy at ARID1A (Fig. 1a). Co-existing PIK3CA mutation was significantly associated with ARID1A mutation, and a majority (61\%) of heterozygous ARID1A tumors also have PIK3CA alterations (Fig. 1a). These data demonstrate that $20 \%$ of endometrioid EC patients are genetically heterozygous for ARID1A mutations and carry PIK3CA alterations.

To induce $\mathrm{CRE}$ in the mouse endometrial epithelium, we utilized LtfCre ( $\mathrm{Tg}(\mathrm{Ltf}-\mathrm{CCre}) 14 \mathrm{Mmul})$. LtfCre induction occurs naturally as females undergo sexual maturity, becoming fully active by 60 days $^{32}$ (Fig. 1b). To investigate the consequence of ARID1A loss in the endometrial epithelium, we bred $\mathrm{LtfCre} \mathrm{C}^{\mathrm{o}+}$ mice to mice with an Arid1afl allele, permitting conditional knockout of ARID1A upon CRE expression (Fig. 1c) ${ }^{30}$ Genotyping by PCR confirmed expression of each allele (Fig. 1d). We observed no gross phenotypes in $\mathrm{LtfCre}^{\mathrm{O} /+}$; Aridlafl/fl mice (Supplementary Fig. 1a). Previously, we found (Gt)R26Pik3$\mathrm{ca}^{* H 1047 R}$ to be a potent driver of epithelial ovarian tumors when combined with Arid1aflfl 30. (Gt)R26Pik3ca ${ }^{*} H 1047 R$ provides conditional expression of the oncogenic PIK3CA ${ }^{\mathrm{H} 1047 \mathrm{R}}$ mutation (Fig. 1c $)^{33}$. No gross phenotypes were observed in $\mathrm{LtfCre} \mathrm{O}^{0 /+} ;(\mathrm{Gt})$ R26Pik3ca ${ }^{*} H 1047 R$ (Supplementary Fig. 1a), as previously described in the endometrial epithelium ${ }^{34}$. Therefore, we bred LtfCre mice with mice harboring $(G t) R 26 P i k 3 c a^{*} H 1047 R$, Arid1afl, and Arid1aV1068G (DNA-binding domain defective ARID1A mutant, Fig. 1c) ${ }^{20}$ to develop an allelic series with increasing ARID1A mutational burden in the endometrial epithelium.

Abnormal vaginal bleeding is a prominent symptom of endometrial dysfunction in humans. LtfCre ${ }^{0 /+}$; $(\mathrm{Gt}) \mathrm{R} 26 \mathrm{Pik3-}$ $\mathrm{ca}^{*}{ }^{*} 1047 R$; Arid1 $a^{f l / f l}$ mice were sacrificed after a median age of 14 weeks due to vaginal bleeding and uterine tumors (Fig. 1e, g). Surprisingly, homozygous ARID1A loss was not required for vaginal bleeding, as $\mathrm{LtfCre} \mathrm{O}^{\mathrm{O}+}$; (Gt)R26Pik3ca ${ }^{*} \mathrm{H} 1047 \mathrm{R}$; Arid1afl/+ mice developed endometrial lesions and vaginal bleeding (Fig. 1e, g). For both LtfCre $e^{0 /+}$; (Gt)R26Pik3ca $\mathrm{ca}^{*} 1047 \mathrm{R}$; Arid1afl/+, and $\mathrm{LtfCre}^{0 /+}$; (Gt)R26Pik3ca ${ }^{* H 1047 R}$; Arid1afl/V1068G mice, median uterus weight, and survival were not significantly different from $\mathrm{LtfCre}^{0 /+}$; (Gt)R26Pik3ca ${ }^{\star} H 1047 R ;$ Arid1aflfl (Fig. 1f, g). ARID1A loss and PI3K pathway activation (via phospho-S6 ribosomal protein, P-S6, expression) were determined by immunohistochemistry, while Cytokeratin 8 (KRT8) labeled the endometrial epithelium (Fig. 1h and Supplementary Fig. 1b). $\mathrm{LtfCre}^{0 /+}$; (Gt) R26Pik3ca ${ }^{*} H 1047 R ;$ Aridlafli+, LtfCre ${ }^{0 /+} ;(\mathrm{Gt}) \mathrm{R} 26 \mathrm{Pik} 3 \mathrm{ca}^{*} \mathrm{H1047R}$; Arid1afl/V1068G, and LtfCre ${ }^{0 /+}$; (Gt)R26Pik3ca ${ }^{*} H 1047 R$; Arid1 afl/fl showed evidence of widespread atypical endometrial hyperplasia and nuclear atypia, including glandular crowding and abnormal cytologic features (Fig. 1h and Supplementary Fig. 1b). Endometrial tumors were moderately to poorly differentiated, with areas of solid and cribiform architecture (Fig. 1h). In one mouse, we observed visible lung metastasis (Supplementary Fig. 1c), a site of metastasis in some EC patients. In the $\mathrm{LtfCre}^{0 /+}$; (Gt) R26Pik3 $\mathrm{ca}^{*} \mathrm{H1047R}$; Arid1afl/fl endometrial epithelium we observed downregulation of estrogen receptor- $\alpha$ (ESR1) and loss of the progesterone receptor, suggesting changes to steroid hormone regulation (Supplementary Fig. 2a). Impaired steroid hormone regulation indicates poor prognosis in $\mathrm{EC}^{35}$. 

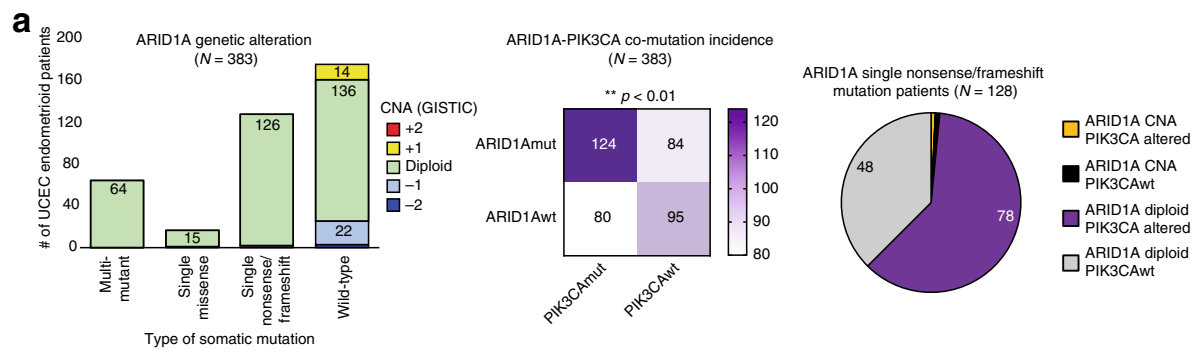

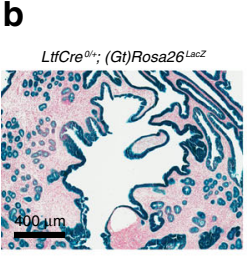

C (Gt)Rosa26 $6^{\text {P.K3Ca'H1047R }}$

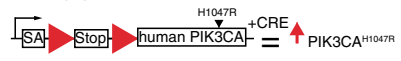
Arid $1 a^{\prime \prime}$
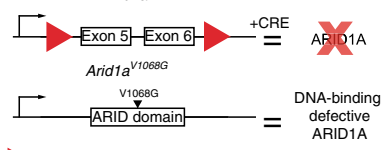

$>$ loxP site

e
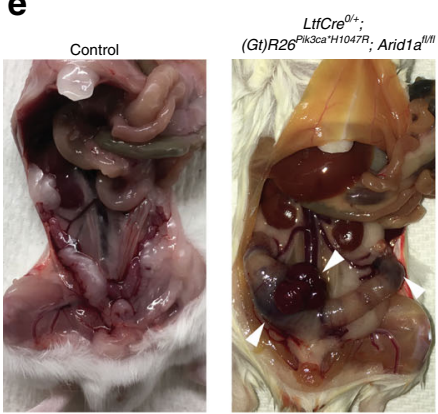

h

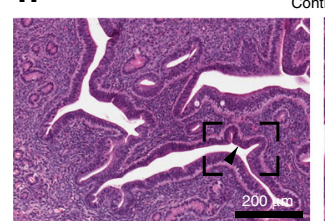

Contro
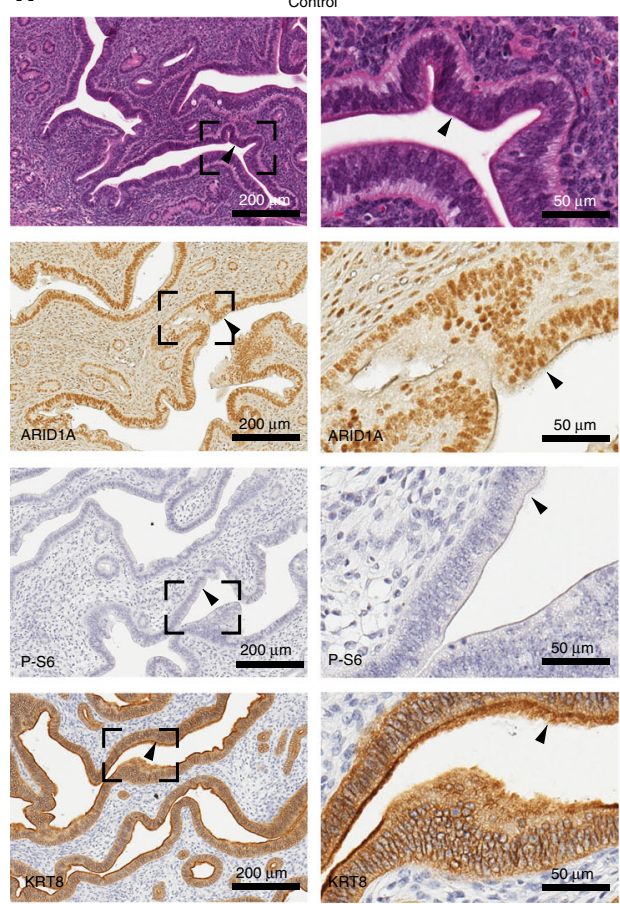

f

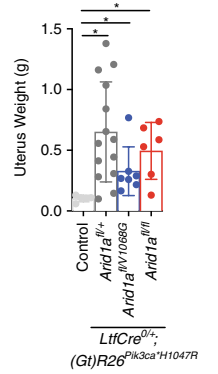

d

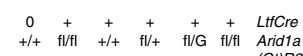

$=_{300 \mathrm{bp}}^{500 \mathrm{pp}}$ - ニニニニ-LHCre

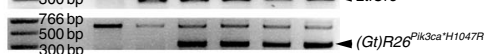

$-300 \mathrm{bp}$
$-50 \mathrm{bp}$

$=-300 \mathrm{bp}-150 \mathrm{bp}-2=-$ Arid1 $^{\prime \prime}$

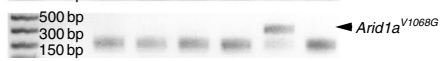

g

LtfCre ${ }^{0 /+}$;

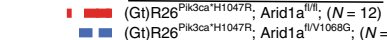

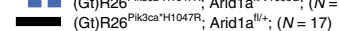

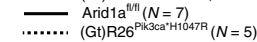

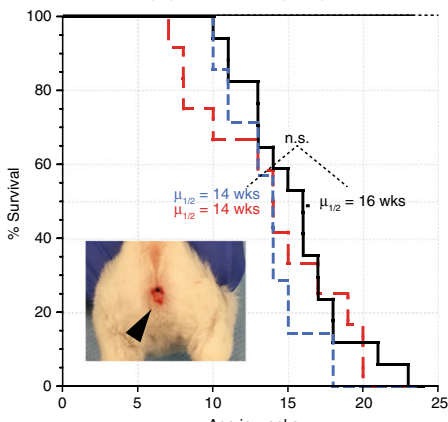

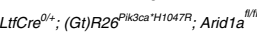
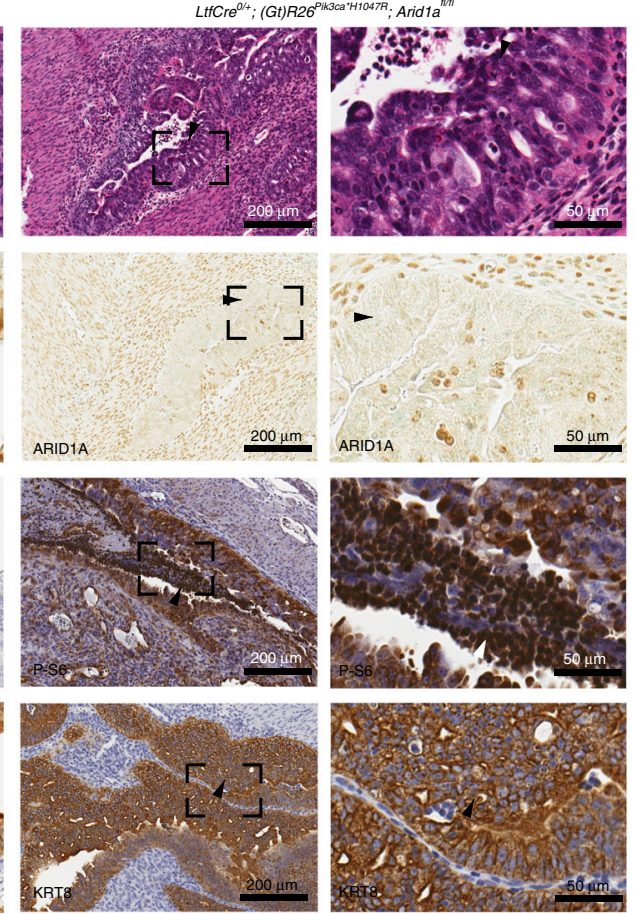

Mutant endometrial epithelium show hallmarks of EMT. To profile in vivo gene expression changes in mutant endometrial epithelium at an early stage of transformation, we devised an enzymatic digestion and magnetic isolation protocol to positively enrich epithelial populations (Fig. 2a). Endometrial epithelial cells express EPCAM (Fig. 2b), and EPCAM expression is not altered

in the hyperplastic endometrium of $\mathrm{LtfCre}{ }^{0 /+}$; (Gt)R26Pik3$c a^{*}$ H1047R; Aridlafl/fl mice (Fig. 2c). Following positive selection, we analyzed purified populations by flow cytometry and observed no significant difference in purity between genotypes (Supplementary Fig. 3a, b). We isolated RNA from control and $L t f C r e^{O /+}$; (Gt)R26Pik3ca H1047R; Arid1 $a^{f l / f l}$ mice. Purified LtfCre $e^{0 /+}$; (Gt) 
Fig. 1 Development of genetic mouse models representing an allelic series of ARID1A mutations in the endometral epithelium. a UCEC endometrioid patient ARID1A alteration status and co-incidence with PIK3CA mutation, taken from TCGA-UCEC dataset. $\mathbf{b}$ LacZ expression (blue) is specific to the endometrial epithelium. Sections were counter-stained with nuclear fast red (scale bar $=400 \mu \mathrm{m}$ ). c Diagram of mutant alleles utilized in this study. $\mathbf{d}$ PCR genotyping results to detect $\mathrm{LtfCre} / \mathrm{O},(\mathrm{Gt}) \mathrm{R} 26 \mathrm{Pik} 3 \mathrm{ca}^{*} \mathrm{H1047R}$, Arid1afl, and Arid1aV1068G. e Representative gross images of mice at time of sacrifice due to vaginal bleeding. White arrows indicate tumors. Size of uterine tumor varies within genotype at time of sacrifice. $\mathbf{f}$ Weight of semi-dry mouse uterus by

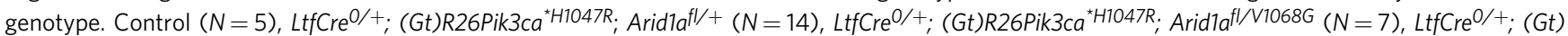

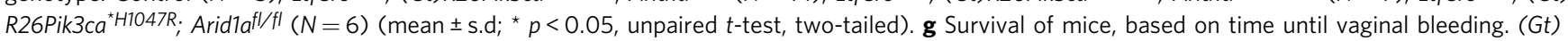

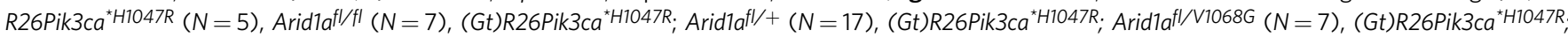
Arid1afl/fl $(N=12)$. Mice succumb to vaginal bleeding (sample image inset) at a median $\left(\mu_{1 / 2}\right)$ of 16 weeks $\left(L t f C r e O /+;(G t) R 26 P i k 3 c a^{*} H 1047 R\right.$ Arid1afl/fl) or

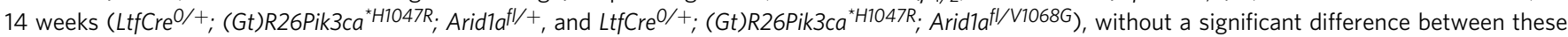
genotypes. LtfCre ${ }^{0 /+}$ mice harboring Arid1afl/fl or (Gt)R26Pik3ca ${ }^{*} H 1047 R$ alone did not develop vaginal bleeding. $\mathbf{h}$ H\&E staining and IHC for ARID1A, P-S6 and KRT8 $(N \geq 2)$ of the endometrium at $5 \times($ scale bar $=200 \mu \mathrm{m})$ and $20 \times($ scale bar $=50 \mu \mathrm{m})$ magnification, with $\times 20$ magnifications representing portion panel to the right surrounded by black box. ARID1A expression is lost in the endometrial epithelium of $\mathrm{LtfCre}^{\mathrm{O} /+}$; (Gt)R26Pik3ca ${ }^{*} \mathrm{H} 1047 \mathrm{R}$; Arid1afl/fl mice. P-S6 is shown as marker of AKT pathway activation; KRT8 as a marker of endometrial epithelium arrows indicate endometrial epithelium

R26Pik3ca ${ }^{*} H 1047 R ;$ Arid1afl/fl cells showed significantly reduced ARID1A messenger RNA (mRNA) expression (Fig. 2d). These samples were processed for RNA-seq, from which we observed 3481 differentially expressed genes between control and $\mathrm{LtfCre}^{0 /+}$; (Gt)R26Pik3ca ${ }^{* H 1047 R ; ~ A r i d l a t l f l ~} \quad(\mathrm{FDR}<0.05) \quad$ (Supplementary Fig. 3c). Using stringent criteria (FDR $<10^{-5}$, twofold change), we identified a gene signature of 517 differentially expressed genes (Supplementary Fig. 3d). We found overlap between $\mathrm{LtfCre}^{0 /+}$; (Gt)R26Pik3ca ${ }^{* H 1047 R ;} \quad$ Aridlafl/fl, LtfCre ${ }^{0 /+}$; (Gt)R26Pik3$\mathrm{ca}^{* H 1047 R ; A r i d 1 a^{f l /+} \text { and LtfCre }}{ }^{0 /+}$; (Gt)R26Pik3ca ${ }^{*}$ H1047R; Arid1afl/V1068G, including 963 genes differentially expressed in all genotypes relative to control (Supplementary Fig. 3e-g).

We performed Gene Set Enrichment Analyses (GSEA) on differentially expressed genes $(\mathrm{FDR}<0.05)$ in $\mathrm{LtfCre}^{0 /+}$; $(\mathrm{Gt})$ R26Pik3ca ${ }^{* H 1047 R ; ~ A r i d 1 a f l f l ~ e n d o m e t r i a l ~ e p i t h e l i a l ~ c e l l s ~ a n d ~}$ identified EMT as the top dysregulated pathway using hallmark pathway enrichment (Fig. 2e). Mesenchymal-marker overexpression in EC correlates with poor prognosis ${ }^{36}$, which is consistent with several Gene Ontology (GO) pathways related to cell motility, migration and adhesion that were identified (Fig. 2f), further suggesting EMT as a key dysregulated pathway in the LtfCre ${ }^{0 /+}$; (Gt)R26Pik3ca ${ }^{*} H 1047 R ;$ Arid1a fl/fl endometrial epithelium. Recently, Mak et al. ${ }^{37}$ identified a patient-derived EMT signature of 77 genes across 11 cancer types. This gene signature was significantly enriched by GSEA in $\mathrm{LtfCre}^{0 /+}$; $(\mathrm{Gt}) \mathrm{R} 26 \mathrm{Pik3-}$ $\mathrm{ca}^{* H 1047 R ;}$; Aridlafl/fl vs. control, and in ARID1A mutant UCEC patients vs. ARID1A wild-type patients (NES $=1.72$ and 1.88, respectively) (Fig. 2g), and contained 33 genes that were differentially expressed in mutant mouse endometrial cells (Fig. 2h).

EMT is characterized by the loss of cell adherens junctions, tight junctions and apical-basal polarity ${ }^{38}$. In LtfCre $^{0 /+} ;$ Arid $1 a^{f l / f l}$ mice, we observed reduced CLDN10 and tight junction protein-1 (ZO-1) expression by immunofluorescence (IF), while expression of ICAM-1 was induced, indicating impaired tight junctions (Supplementary Fig. 4a-d). ZO-1 expression was partially restored in $\mathrm{LtfCre} \mathrm{O}^{0 /+}$; (Gt)R26Pik3ca ${ }^{*} \mathrm{H1047R}$; Arid1 afl/fl (Supplementary Fig. 4a). LtfCre ${ }^{0 /+}$; Aridlafl/fl endometrium has high expression of Cleaved Caspase-3 (CASP3), indicating increased apoptosis in the absence of PIK3CA ${ }^{\text {H1047R }}$ (Supplementary Fig. 4e). Expression of mesenchymal-marker VIM (Vimentin) and EMT transcription factor SNAI2 (Slug) were observed in both $\mathrm{LtfCre}^{0 /+}$; Arid1a fl/fl and LtfCre ${ }^{0 /+}$; (Gt)R26Pik3ca ${ }^{*} \mathrm{H1047R}$; Aridlaflfl mutant endometrial epithelium, indicating a shift towards a mesenchymal phenotype (Supplementary Fig. 4f, g). CDH1 (E-Cadherin) mislocalization was observed in mutant endometrial epithelium, suggesting alterations in epithelial adherens junctions (Supplementary Fig. 4h). These data suggest that the EMT phenotype observed in $\mathrm{LtfCre}^{\mathrm{O} /+}$; $(\mathrm{Gt})$
$R 26 P i k 3 c a^{*} H 1047 R ;$ Arid1aflfl endometrial epithelium are driven primarily by ARID1A loss.

Mouse gene signature identifies invasive patient population.

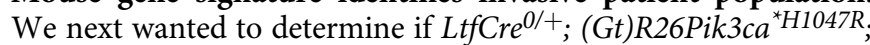
Arid $1 a^{f l / f l}$ gene expression patterns resembled human disease. We utilized mutation and RNA-seq expression data from the TCGAUCEC dataset with single-sample GSEA (ssGSEA) to rank UCEC patient endometrioid tumors with gene expression patterns similar to our mouse model. We segregated the upper (similar to mouse) and lower (dissimilar to mouse) quartiles of patients based on human orthologs of our gene signature (Fig. 3a). Upper quartile UCEC patients display concordant expression changes for $74 \%$ of genes within the $\mathrm{LtfCre}{ }^{0 /+}$; (Gt)R26Pik3 $\mathrm{ca}^{*} \mathrm{H} 1047 \mathrm{R}$; Arid1afl/fl gene signature relative to lower quartile patients (Fig. 3b). Upper quartile patients show upregulation of EMT, Interferon gamma (IFN $\gamma$ ), Notch and P53 signaling pathways, and downregulation of the unfolded protein response (UPR) (Fig. 3c). We confirmed downregulation of GRP94 and GRP78, two proteins critical to the UPR, in the $\mathrm{LtfCr} \mathrm{e}^{\mathrm{O}+}$; $(\mathrm{Gt}) \mathrm{R} 26 \mathrm{Pik} 3$ $c a^{*}{ }^{*} 1047 R ; A r i d 1 a^{f l / f l}$ endometrial epithelium in vivo by IHC and IF (Supplementary Fig. 2b, c). When comparing ARID1A mutant and wild-type UCEC patients, we also identified upregulation of the EMT pathway (Fig. 3d).

Clinical staging of endometrial cancer is determined by invasion into surrounding tissue, including the myometrium, cervix, vagina, bladder, and distant metastasis ${ }^{2}$. Upper quartile patients were diagnosed with advanced clinical stage relative to all UCEC patients, with significantly more stage III and stage IV patients ( $p<0.01$, Chi-squared) (Fig. 3e). Furthermore, upper quartile patients had significantly more invasion than lower quartile patients $(p<0.05$, unpaired Mann-Whitney $U$, twotailed) (Fig. 3f). These data suggest that endometrial cells from LtfCre ${ }^{0 /+} ;(G t) R 26 P i k 3 c^{*} a^{*} 1047 R ;$ Arid1a fl/fl mice are representative of UCEC patients with advanced stage, invasive tumors.

ARID1A loss increases promoter accessibility in vivo. To gain insight into chromatin accessibility alterations that may drive the observed gene expression changes, we performed ATAC-seq (Assay for Transposase-Accessible Chromatin) ${ }^{39}$ on antiEPCAM-purified cells. In general, the peaks were broader in LtfCre ${ }^{O /+}$; (Gt)R26Pik3ca ${ }^{*}$ H1047R; Arid1afl/fl cells compared to cells from control mice $\left(p<10^{-15}\right.$, unpaired Mann-Whitney $U$, two-tailed), potentially indicating greater chromatin accessibility in mutant cells (Fig. 4a, b). Among differentially accessible peaks (FDR < 0.20), 2053 showed decreased accessibility in $\mathrm{LtfCre}^{\mathrm{O/+}}$; (Gt)R26Pik3ca H1047R; Arid1aflfl mice, while 1429 showed increased accessibility, suggesting a global trend toward 
a

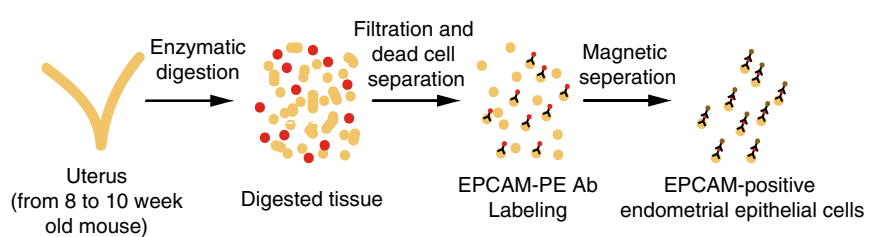

b

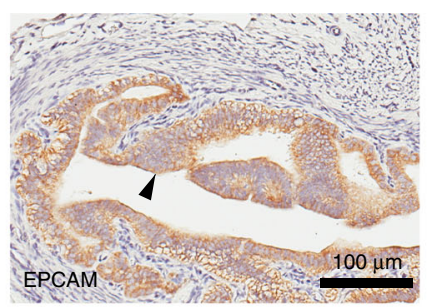

C
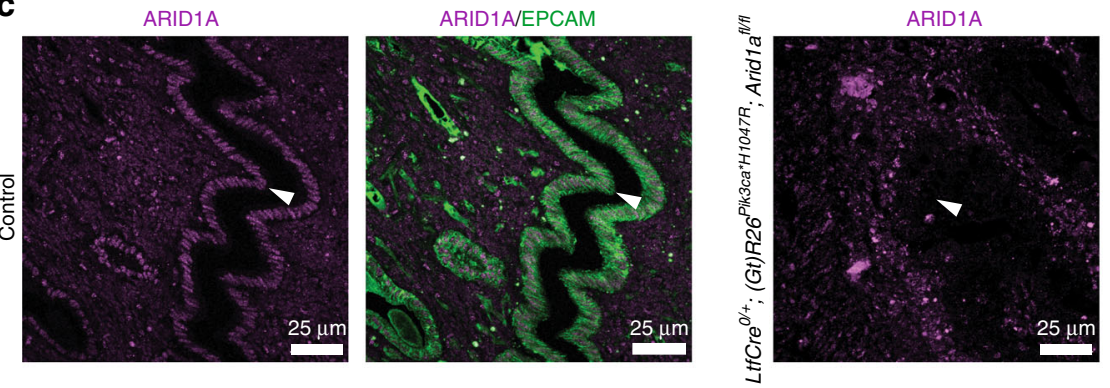

Hallmark enrichment (orthologs)

d e f
Epithelial mesenchymal transition KRAS signaling (up) Interferon gamma response Inflammatory response Estrogen response early IL2 STAT5 signaling Allograft rejection

UV response (down) Interferon alpha response Angiogenesis

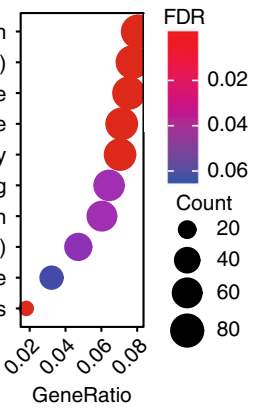

ARID1A/EPCAM

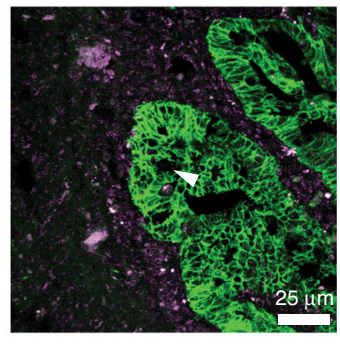

GO enrichment

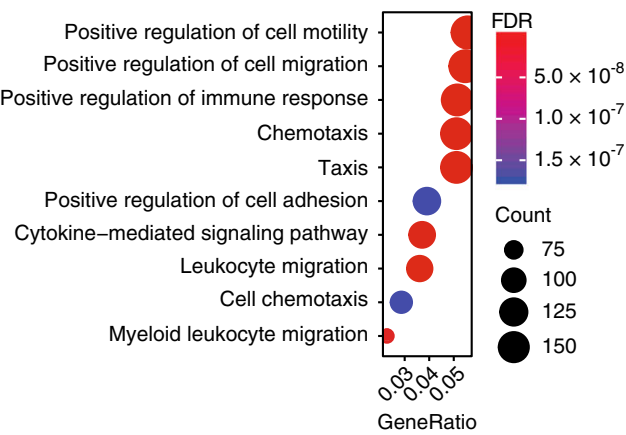

g

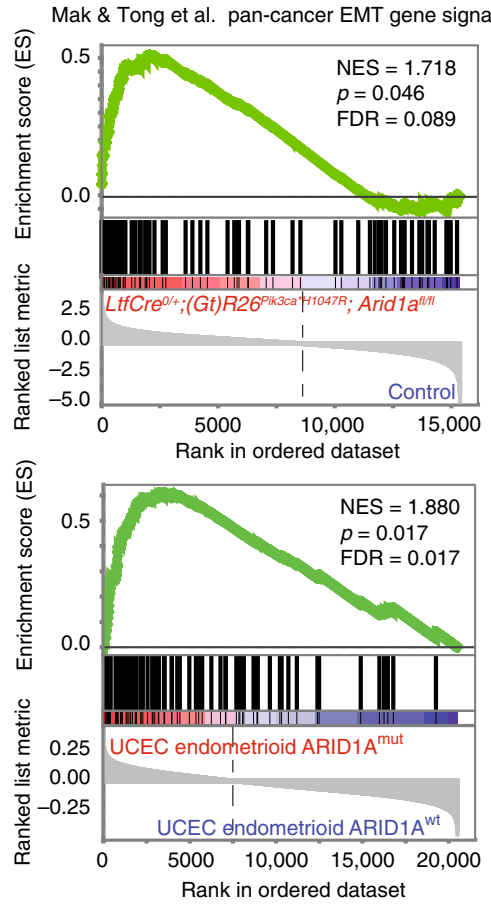

h

Mak \& Tong et al. pan-cancer EMT gene signature

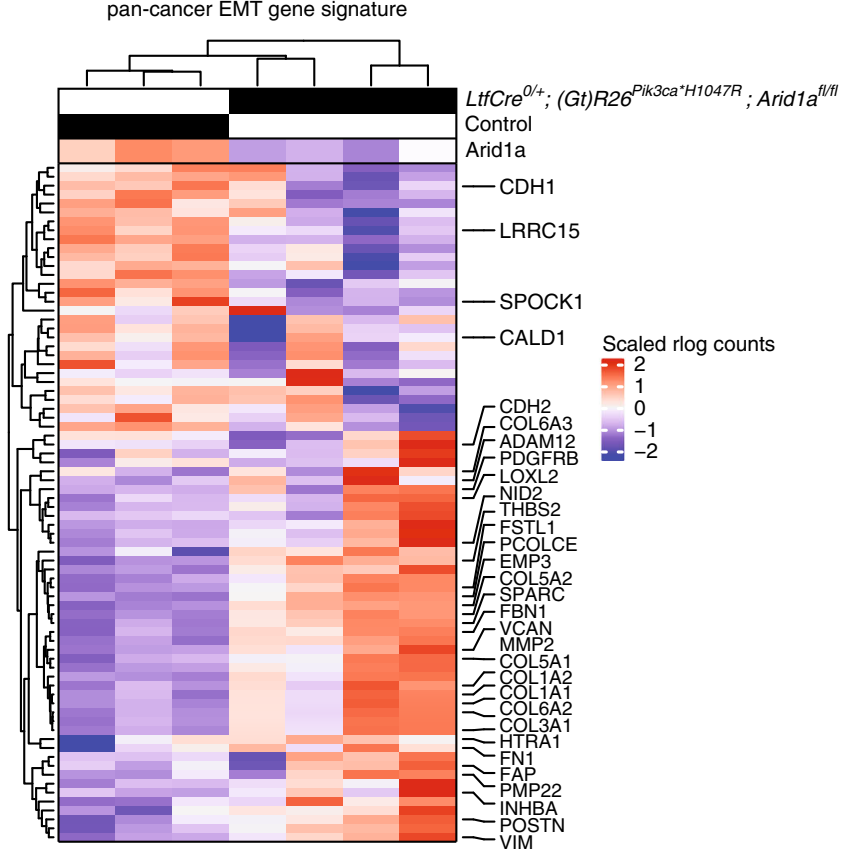

decreasing accessibility (Fig. 4c). Primarily, differentially accessible peaks represented mononucleosome fragments (Fig. 4d). Despite the trend toward decreased accessibility, among promoters (defined as regions $\pm 3 \mathrm{~kb}$ to transcription start sites or TSS) we observed significant increases in accessibility $\left(p<10^{-72}\right.$, Chi-squared) (Fig. 4e), with 470 promoter peaks increasing in accessibility and 179 decreasing (Fig. 4f). Genomic repeat elements trended toward decreased accessibility (80\% decreasing), accounting for a global trend toward decreasing accessibility (Fig. 4f). Among peaks with increased accessibility, CpG islands, promoters and $5^{\prime}$ UTR were the top enriched genomic features (Fig. 4g). Differentially accessible peaks, including promoter 
Fig. 2 RNA-seq analysis of EPCAM-positive endometrial epithelial cells isolated via magnetic sorting. a Schematic of EPCAM isolation using anti-EPCAM-

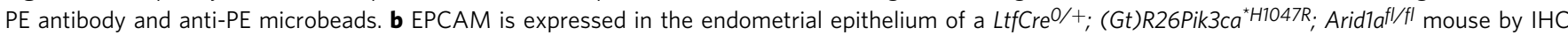
$(N=3)$. Arrows indicate endometrial epithelium (scale bar $=100 \mu \mathrm{m})$. c IF staining of EPCAM and ARID1A in mouse endometrium ( $N \geq 3$ ). Arrows indicate endometrial epithelium (scale bar $=25 \mu \mathrm{m})$. $\mathbf{d}$ qPCR analysis of Aridla gene expression of isolated control $(\mathrm{N}=3$, pooled groups of six mice) and $\mathrm{LtfCre}^{\mathrm{O} /+}$; (Gt)R26Pik3ca H1047R; Arid1afl/fl (mutant) $\left(N=4\right.$, single mice) cells (mean $\pm \mathrm{s} . \mathrm{d}_{;}{ }^{* \star} \mathrm{p}<0.01$, unpaired $t$-test, two-tailed). e, $\mathbf{f}$ Pathway enrichment analysis on human orthologs of differentially expressed genes between LtfCre ${ }^{O /+}$; (Gt)R26Pik3ca ${ }^{*} H 1047 R$; Arid1afl/fl, and control mice (FDR < 0.05; 3481 genes) for mSigDb Hallmark pathways (e) and Gene Ontology (GO) Biological Process terms (f). $\mathbf{g}$ GSEA plots showing significance of Mak et al. pan-

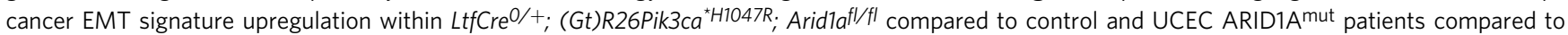
ARID1A $^{\text {wt }}$. $\mathbf{h}$ Hierarchical clustering of 77 genes within the Mak et al. pan-cancer EMT signature between control and mutant purified endometrium. Genes found in the Hallmark EMT pathway, and CDH1, are identified

peaks, were generally located proximal to TSS, with $31.2 \%$ of all peaks located within $10 \mathrm{~kb}$ of a TSS (Fig. 4h, i). We also performed ATAC-seq on EPCAM-purified cells from $\mathrm{LtfCre} \mathrm{C}^{\mathrm{O}+} ;(\mathrm{Gt})$ R26Pik3ca ${ }^{*}$ H1047R; Arid1afl/t and LtfCre ${ }^{0 /+}$; (Gt)R26Pik3$\mathrm{ca}^{*}$ H1047R; Arid1a fl/V1068G, and observed enrichment for differential accessibility among promoters $\left(p<10^{-500}\right)$ (Supplementary Fig. $3 \mathrm{~h}-\mathrm{p}$ ).

Among genes with differentially accessible promoter peaks, EMT appeared as the top enriched pathway (Fig. 4j). We identified significant overlap between differentially accessible promoters and differentially expressed genes $\left(p<10^{-8}\right.$, hypergeometric enrichment) (Fig. 4k). Chromatin accessibility was positively correlated with gene expression $\left(p<10^{-9}\right.$, Spearman) (Fig. 4l). Among these genes, EMT again appeared as a top affected pathway by enrichment analysis (Fig. 4m). Altogether, these data demonstrate that endometrial ARID1A loss and PI3K activation results in increased accessibility at gene promoters and differential accessibility of EMT pathway genes.

ARID1A functionally binds gene promoters. To explore the role of ARID1A loss alone in the regulation of endometrial epithelial chromatin accessibility, we utilized an immortalized human endometrial epithelial cell line, $12 Z^{40}$. Transfection of $12 \mathrm{Z}$ cells with short-interfering RNAs (siRNAs) targeting ARID1A (siARID1A) reduced ARID1A protein expression relative to cells transfected with non-targeting control (siNONtg) (Fig. 5a). Next, we performed ATAC-seq on siARID1A transfected $12 \mathrm{Z}$ cells (Supplementary Fig. 5a-d). ARID1A loss led to a trend toward decreasing chromatin accessibility genome-wide, while chromatin accessibility was significantly increased at promoters $\left(p<10^{-500}\right.$, Chi-squared) (Fig. $5 b$ ). These results recapitulate our findings in vivo, suggesting differential chromatin changes in vivo are driven by ARID1A loss alone.

In order to profile sites of genome-wide ARID1A occupancy, we performed ARID1A ChIP-seq in $12 \mathrm{Z}$ cells. The specificity of the ARID1A ChIP-seq antibody used was validated by coimmunoprecipitation (co-IP) and mass spectrometry (Supplementary Fig. 5e, f). We identified 46,180 unique sites of ARID1A genome-wide occupancy (Fig. 5c). The majority of ARID1A ChIP-seq peaks were less than $1000 \mathrm{bp}$ in width (Fig. 5d) and generally were proximal to TSS, with roughly one-quarter of all peaks being within $10 \mathrm{~kb}$ of the TSS (Fig. 5e, f). ARID1A binding was significantly enriched at promoters (Fig. 5g). Among ARID1A-bound sites, we observed an enrichment of the AP-1 motif, both genome-wide $\left(p<10^{-8170}\right)$ and at promoters $(p<$ $\left.10^{-800}\right)$. ARID1A has been shown to regulate chromatin accessibility at AP-1 motifs ${ }^{41,42}$, and we also observed an enrichment for the AP-1 motif at sites of differential accessibility in vivo and in vitro (Supplementary Fig. $5 \mathrm{~g}$ ), suggesting ARID1A regulation of chromatin at AP-1 sites.

ARID1A-bound promoters were enriched for EMT hallmark genes (Fig. 5i). We also observed significant overlap between ARID1A binding and sites of accessible chromatin, which were positively correlated ( $p<10^{-15}$, Spearman) (Fig. 5j, k). Among differentially accessible promoters, ARID1A was bound to 354 promoters, which increased in accessibility, and 124 promoters, which decreased in accessibility upon ARID1A loss (Fig. 5l).

To further explore the relationship between ARID1A binding and gene expression, we performed RNA-seq on siNONtg and siARID1A treated $12 \mathrm{Z}$ cells. Differentially expressed genes (FDR $<0.0001)$ were significantly enriched for the $\mathrm{LtfCre} \mathrm{e}^{0 /+} ;(\mathrm{Gt})$ R26Pik3ca ${ }^{*}$ 1047R; Arid1 aflfl gene signature $\left(p<10^{-5}\right.$, hypergeometric enrichment) (Fig. 5m). ARID1A promoter binding was significantly enriched in differentially expressed genes with ARID1A knockdown $\left(p<10^{-208}\right.$, hypergeometric enrichment) (Fig. 5n). While ARID1A promoter binding was evenly distributed among upregulated and downregulated genes (Supplementary Fig. 5h), we observed a higher degree of gene upregulation following ARID1A loss among genes with ARID1A binding at the promoter $(p=0.002$, unpaired Mann-Whitney $U$, two-tailed) (Fig. 5o). ARID1A bound, upregulated genes are enriched for EMT pathways (Fig. 5p). ARID1A binding is observed in the promoters of mesenchymal identity genes (Fig. 5q). These data support a mechanistic role for ARID1A in the suppression of mesenchymal gene transcription.

ARID1A loss promotes mesenchymal phenotype. To further interrogate the relationship between ARID1A and PIK3CA in the regulation of the EMT pathway, we again utilized the $12 \mathrm{Z}$ cell line. EMT is regulated by several transcription factors, including SNAI1 (Snail), SNAI2 and TWIST1 (Twist) ${ }^{38}$. Upon ARID1A knockdown by siRNA (siARID1A), we observed upregulation of SNAI1, SNAI2, and TWIST1 protein expression (Fig. 6a). Transfection with PIK3CA ${ }^{\mathrm{H} 1047 \mathrm{R}}$ expression plasmid (pPIK3$\mathrm{CA}^{\mathrm{H} 1047 \mathrm{R}}$ ) led to AKT/mTOR pathway activation, as indicated by phosphorylation of AKT at serine 473 (P-AKT Ser473) (Fig. 6a). In cells transfected with both siARID1A and pPIK3CA ${ }^{\mathrm{H} 1047 \mathrm{R}}$, we observed decreased induction TWIST1 (Fig. 6a). Expression of

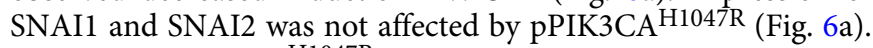
Moreover, pPIK3CA ${ }^{\mathrm{H} 1047 \mathrm{R}}$ induced CDH1 expression (Fig. 6a) and partially rescued the $\mathrm{CDH} 1$ downregulation observed in cells transfected with only siARID1A.

We next performed RNA-seq on cells transfected with

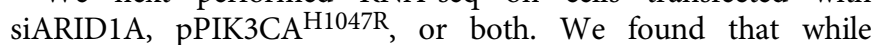
ARID1A loss resulted in differential gene expression of 2565 genes, PIK3CA ${ }^{\mathrm{H} 1047 \mathrm{R}}$ expression resulted in differential gene expression of only 233 genes (FDR < 0.0001) (Fig. 6b). Some genes differentially expressed by PIK3CA $\mathrm{H}^{\mathrm{H} 1047 \mathrm{R}}$ overlapped with siARID1A and siARID1A/PIK3CA ${ }^{\mathrm{H} 1047 \mathrm{R}}$ samples, displaying unique patterns of gene expression (Supplementary Fig. 6). Among Hallmark pathways, we observed siARID1A and PIK3$\mathrm{CA}^{\mathrm{H} 1047 \mathrm{R}}$ convergence on the NFKB pathway, as previously described in ovarian clear cell carcinoma ${ }^{43}$, and the EMT pathway (Fig. 6c). Differentially expressed genes from siARID1A, PIK3$\mathrm{CA}^{\mathrm{H} 1047 \mathrm{R}}$, and siARID1A/PIK3CA ${ }^{\mathrm{H} 1047 \mathrm{R}}$ samples compared to 
a

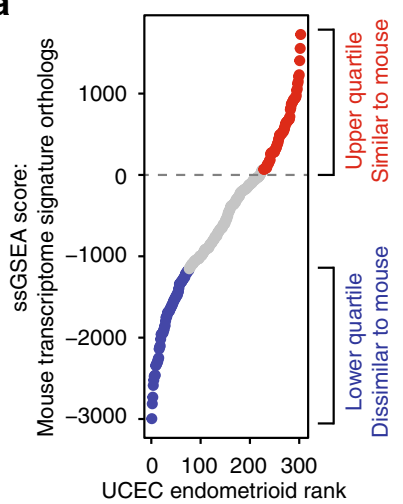

b

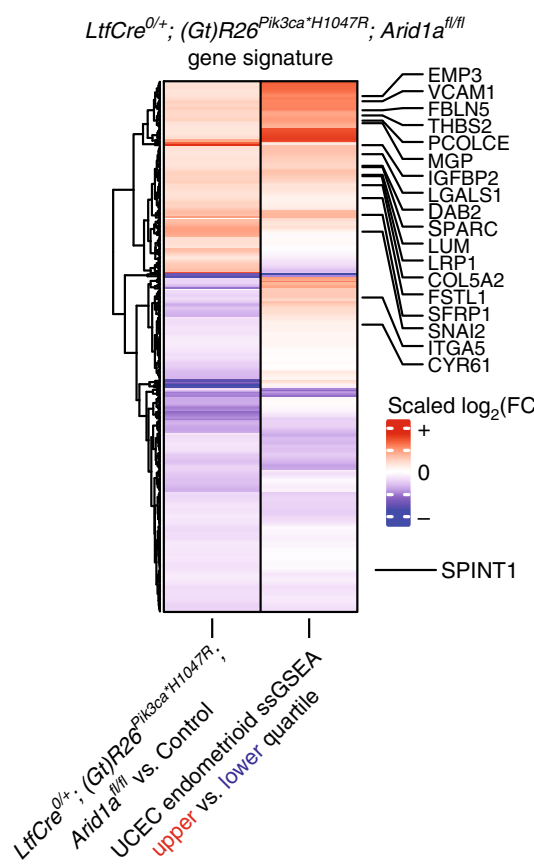

C

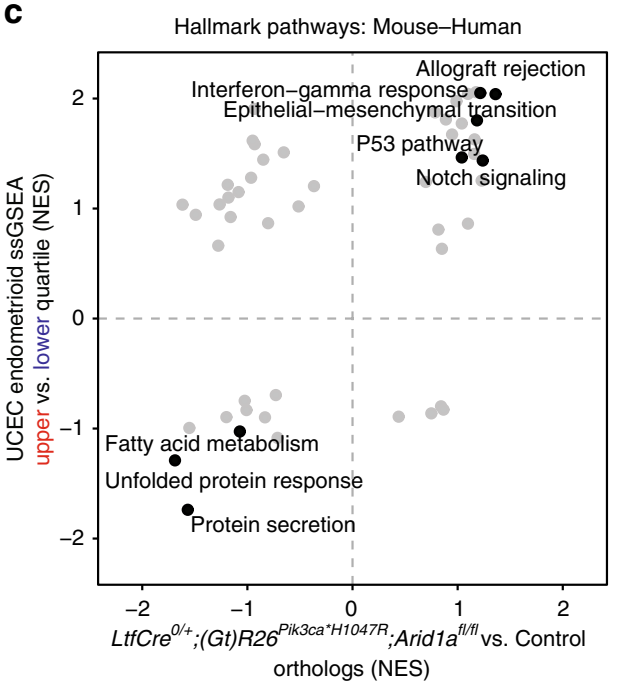

d

dallmark pathways: Human-Human

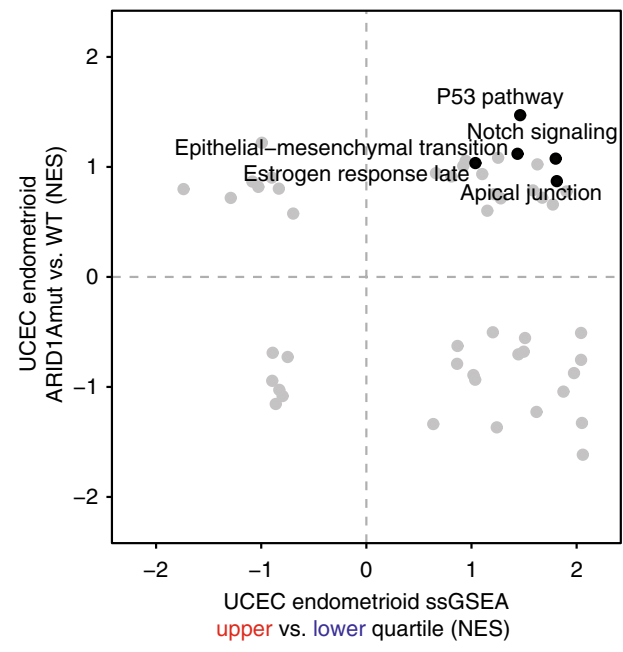

$\mathbf{f}$
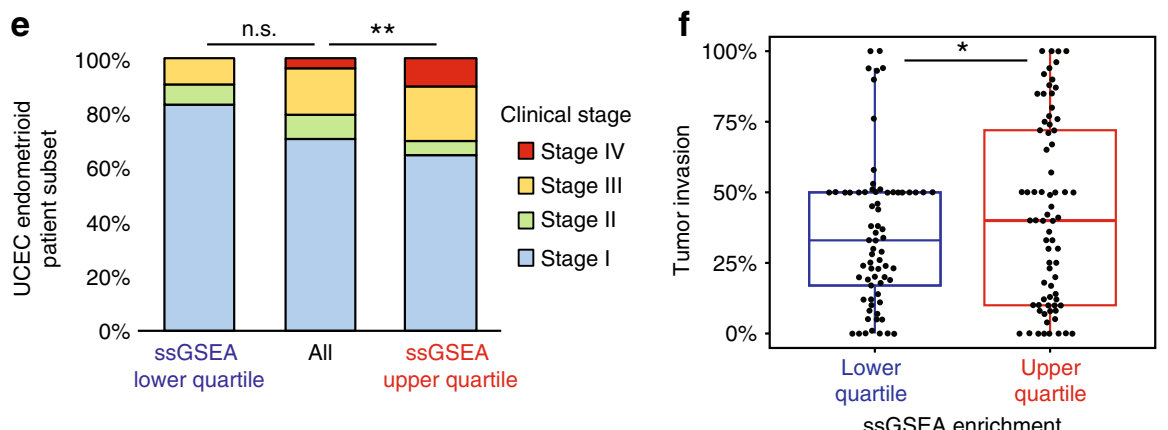

Fig. $3 \mathrm{LtfCre} /++$; (Gt)R26Pik3ca*H1047R; Arid1afl/fl gene signature correlates with invasive patient gene expression. a Distribution of TCGA-UCEC

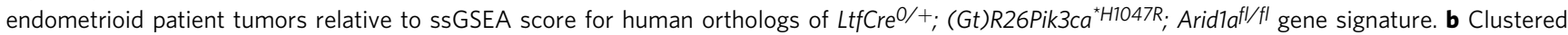
comparison of scaled fold-change values for signature genes between $\mathrm{LtfCre} \mathrm{O}^{+}$; (Gt)R26Pik3ca ${ }^{*} \mathrm{H} 1047 \mathrm{R}$; Arid7a ${ }^{f l / f l}$ vs. control mice and upper vs. lower quartile of UCEC endometrioid patients. EMT genes from Hallmark pathway and Mak and Tong pan-cancer gene signature are identified. c Scatter plot of Hallmark pathway GSEA Normalized Enrichment Scores (NES) from LtfCreO/+; (Gt)R26Pik3ca*H1047R; Arid1afl/fl vs. control (human orthologs) and upper quartile of UCEC endometrioid patients vs. lower quartile. d Scatter plot of Hallmark pathway GSEA NES from upper quartile of UCEC endometrioid patients vs. lower quartile and UCEC endometrioid ARID1Amut (frameshift/truncating alterations) vs. ARID1Awt. e Upper quartile ssGSEA-enriched UCEC endometrioid patients present with higher stage disease relative to all patients ( $p<0.01$, Chi-squared). $\mathbf{f}$ Upper quartile ssGSEA-enriched UCEC endometrioid patients have more invasive tumors relative to lower quartile patients $(p<0.05$, unpaired Mann-Whitney $U$, one-tailed). Box-and-whiskers plotted in the style of Tukey without outliers 
a

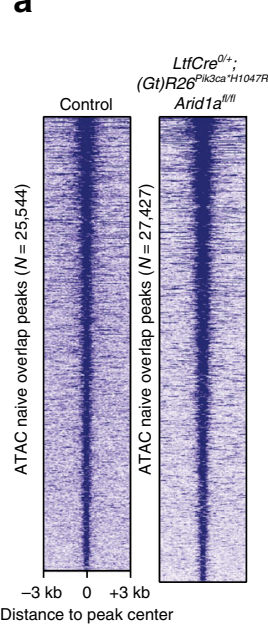

b

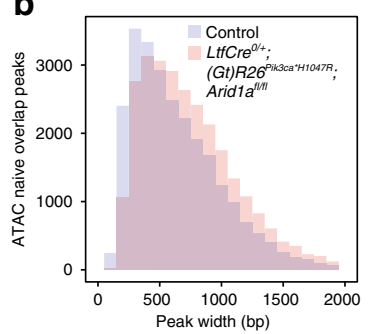

d

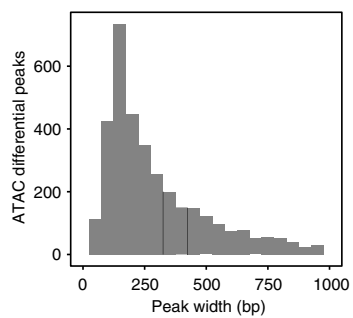

C

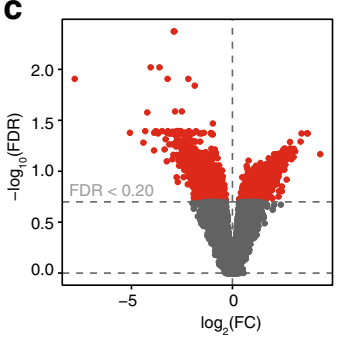

e

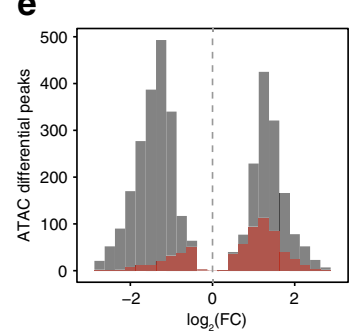

f

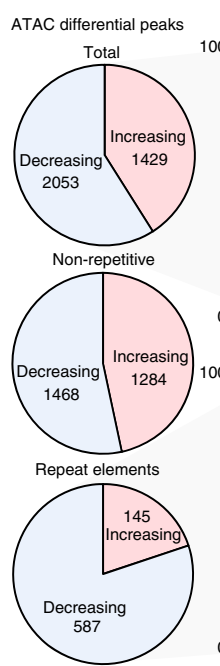

g

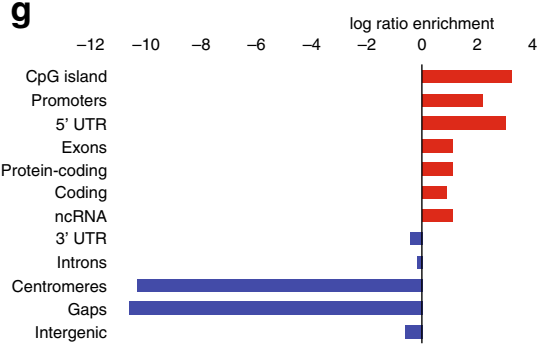

h i

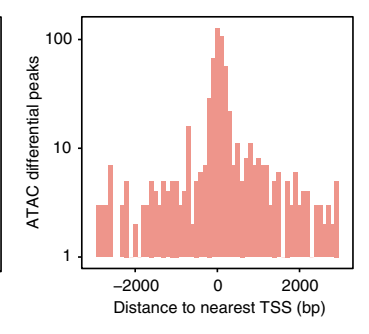

Distance to nearest TSS (kilobases)

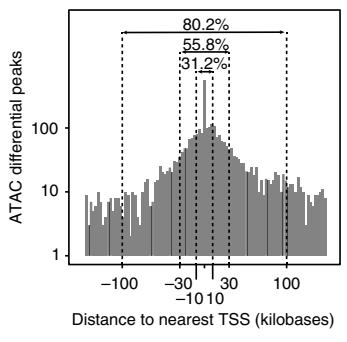

m

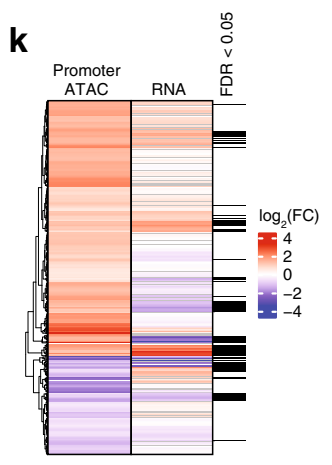

I

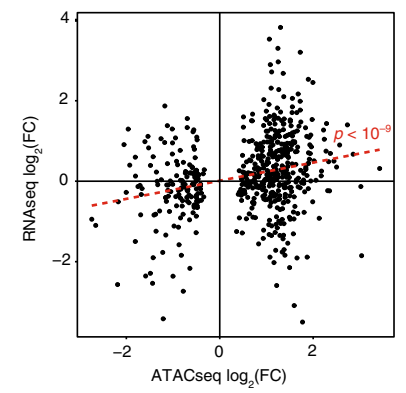

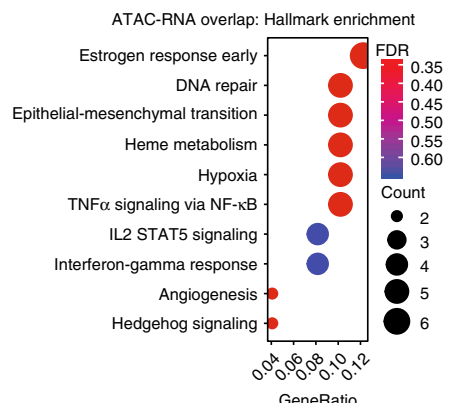

$$
\begin{aligned}
& 35 \\
& 40 \\
& 45 \\
& 50 \\
& 55 \\
& 60 \\
& 4 \\
& 2 \\
& 3 \\
& 4 \\
& 5 \\
& 6
\end{aligned}
$$

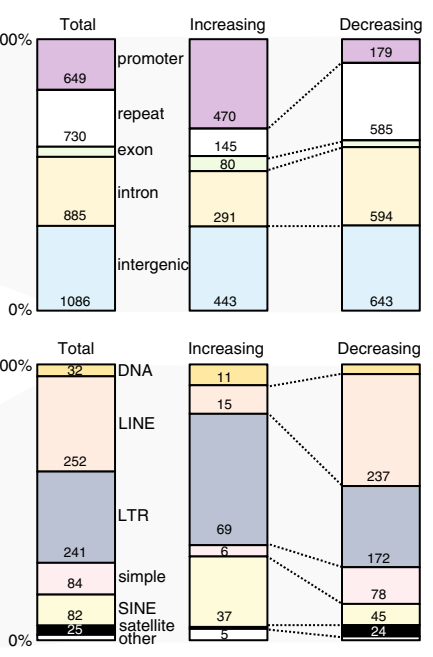

j

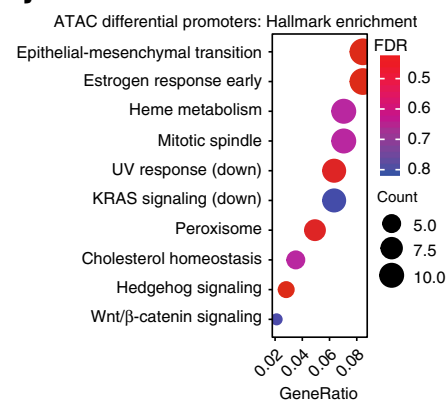

Fig. 4 ATAC-seq analysis of differentially accessible chromatin in $L t f C r e{ }^{O /+}$; (Gt)R26Pik3ca ${ }^{*} H 1047 R$; Arid1a ${ }^{f l / f l}$ endometrial epithelium. a ATAC-seq read density heatmap from naive overlapping peaks of control and $L t f C r e O /+$; (Gt)R26Pik3ca ${ }^{\star} H 1047 R$; Arid1afl/fl EPCAM-positive cells, ranked by total intensity. Reads are centered on the middle of the accessible peak $\pm 3 \mathrm{~kb}$. Control $\left(N=2\right.$, pooled groups of six mice) and $L t f C r e 0 /+;(G t) R 26 P i k 3 \mathrm{ca}^{*} H 1047 R$; Arid1afl/fl $\left(N=2\right.$, single mice). b Peak width distributions of control and $L t f C r e{ }^{O /+} ;(G t) R 26 P i k 3 c a^{*} H 1047 R$; Arid1al/fl ATAC-seq peaks, which are significantly different $\left(p<10^{-15}\right.$, unpaired Mann-Whitney $U$, two-tailed). c Volcano plot for differential accessibility of ATAC-seq peaks between control and LtfCre ${ }^{0 /+}$; (Gt) R26Pik3ca*H1047R; Arid1afl/fl cells. Red points represent significant peaks (FDR $<0.20$ ). d Peak width distribution of differentially accessible peaks.

e Magnitude distribution of differentially accessible peaks separated by total peaks (gray) and promoter peaks (red, within $3 \mathrm{~kb}$ of TSS). $\mathbf{f}$ Detailed peak annotation of increasing and decreasing differentially accessible regions for total, non-repetitive and repetitive peaks based on genome annotation.

g Enrichment for significant genomic features among differentially accessible peaks, ranked by $p$-value. Enrichment ratio is calculated by bp of feature in ATAC peak set compared to background genome. $\mathbf{h}$ Histogram of all differential ATAC peaks depicting distance to nearest TSS. Percent of peaks found within $+/-10,30$, or $100 \mathrm{~kb}$ of the TSS are shown. $\mathbf{i}$ Histogram of differential ATAC promoter peaks depicting distance to nearest TSS. $\mathbf{j}$ mSigDb Hallmark pathway enrichment of genes with differentially accessible promoter peaks. $\mathbf{k}$ Differentially accessible promoter peak clustering based on direction and magnitude of change in gene expression and promoter accessibility. Black bars indicate significant differential gene expression by RNA-seq (FDR < 0.05). I Scatter plot depicting the relationship between direction and magnitude of change in accessibility and gene expression for differential promoter peaks. Accessibility and expression were significantly correlated $\left(r_{\mathrm{s}}=0.26, p<10^{-9}\right.$, Spearman). $\mathbf{m}$ mSigDb Hallmark pathway enrichment of overlapping differentially accessible promoters and differentially expressed genes

controls were enriched for the $L t f C r e^{0 /+}$; (Gt)R26Pik3ca ${ }^{*} H 1047 R$; Arid1 $a^{f l / f l}$ gene signature and the Mak and Tong pan-cancer EMT gene signature (Fig. 6d). For genes found in the Mak and Tong signature and the hallmark EMT pathway, we identified an antagonistic relationship between siARID1A and PIK3CA ${ }^{\mathrm{H} 1047 \mathrm{R} \text {, }}$ such that gene expression changes observed in siARID1A samples were reduced in siARID1A/PIK3CA ${ }^{\mathrm{H} 1047 \mathrm{R}}$ samples (Fig. 6e, f).

To further explore the antagonistic relationship between ARID1A loss and PIK3CA $\mathrm{H}^{\mathrm{H} 1047 \mathrm{R}}$, we identified a unique group of genes at the intersection between differentially 
a

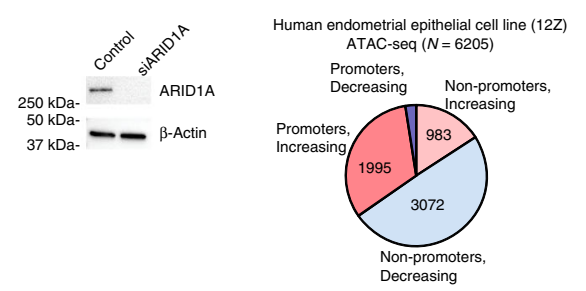

f

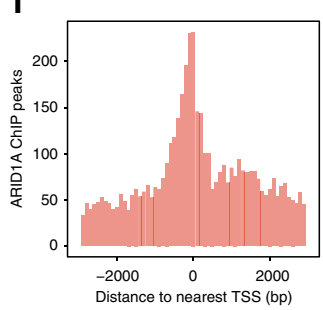

g ARIDIA ChPP Pog ratio enrichment Promoters Promoters
$\mathrm{CPG}$ island CPG island
$5^{\prime}$ UTR

$5^{\prime}$ UTR
IRNA

rRNA
SnRNA

SnRNA
ncRNA
Pseudo

Pseudo
TTS

3' UTR

Coding
Exons

In-coding
Introns

j

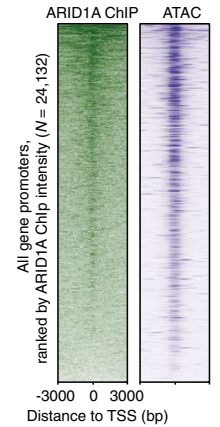

Intergenic

K I $\underset{\substack{\text { ARIDIA-bound } \\ \text { gene promoters }(N=4653)}}{\text { I }}$

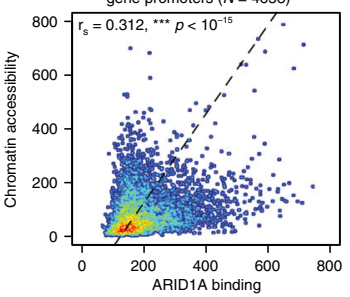

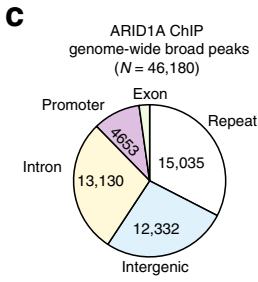

d

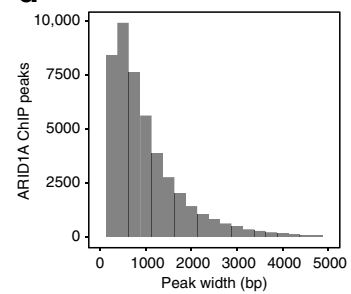

h

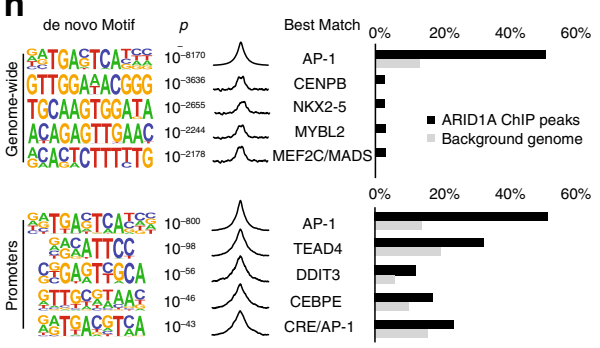

e

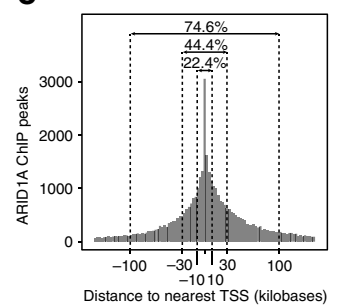

i I
$\begin{aligned} & \text { ARID1A-bound } \\ & \text { promoters }\end{aligned}$
accessidibility decreasing
$(N=150)$

promoters
$(N=3843)$

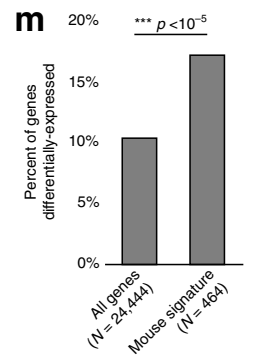

n

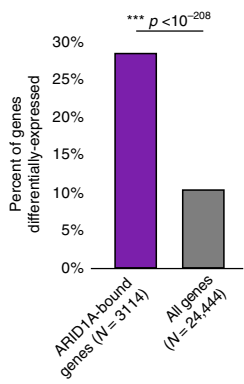

p

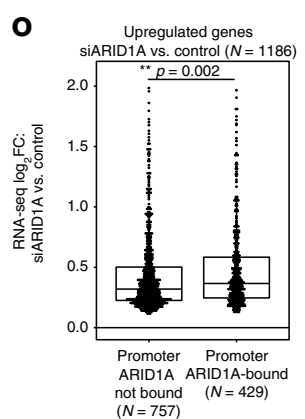

siARID1A vs. Control (FDR < 0.0001)

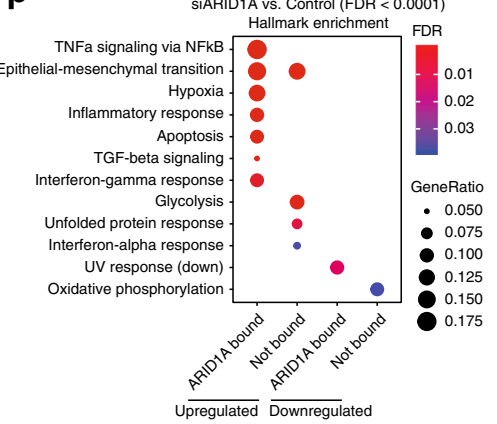

q 0.3 - chr5: 122,075,249-122,080,248

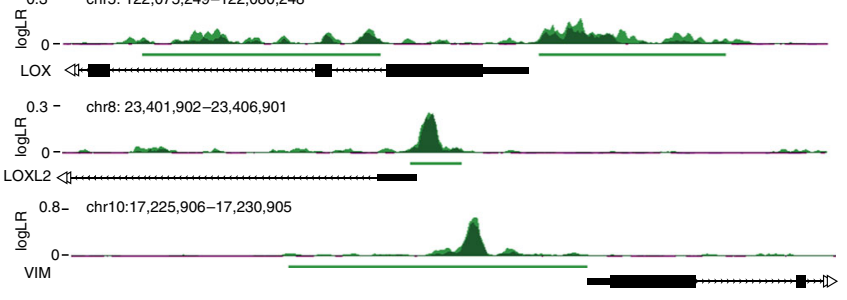

Fig. 5 ARID1A binding is associated with accessibility and differential gene expression driven by ARID1A loss in human endometrial epithelial cell line. a Western blot of ARID1A expression in siRNA-treated $12 Z$ cells. $\beta$-Actin was used as endogenous control. $\mathbf{b}$ Annotation of differentially accessible ATAC peaks (FDR < 0.05) from $12 Z$ siARID1A, separated into fractions by directionality and promoter vs. non-promoter. Significant association $\left(p<10^{-500,}\right.$ Chisquared) between increasing accessibility and promoter status. c Annotation of ARID1A ChIP peaks in wild-type $12 Z$ cells. $\mathbf{d}$ Peak width distribution of ChIP peaks. e Histogram of all ChIP peaks depicting distance to nearest TSS. Percent of peaks found within $+/-10,30$, or $100 \mathrm{~kb}$ of the TSS are shown. $\mathbf{f}$ Histogram of ChIP promoter peaks depicting distance to nearest TSS. $\mathbf{g}$ Enrichment for significant genomic features among ChIP peaks, ranked by $p$-value. Enrichment ratio is calculated by bp of feature in ChIP peak set compared to background genome. $\mathbf{h}$ de novo Motif enrichment of ChIP peaks genome-wide and at promoters. i mSigDb Hallmark pathway enrichment of genes with ChIP promoter peaks. j Read density heatmap of ARID1A ChIP-seq and ATAC-seq (control) at all gene promoters $(N=24,132)$, ranked by signal intensity for ARID1A ChIP-seq. $\mathbf{k}$ Scatter plot depicting correlation between ARID1A binding and chromatin accessibility $\left(r_{\mathrm{s}}=0.312, p<10^{-15}\right.$, Spearman). I Proportional Euler diagram of overlap between ARID1A binding, decreasing and increasing

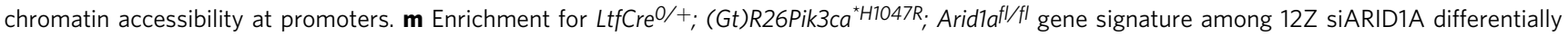
expressed genes $\left(p<10^{-5}\right.$, hypergeometric enrichment). $\mathbf{n}$ Enrichment of ARID1A binding at 12Z siARID1A differentially expressed genes ( $p<10^{-208}$, hypergeometric enrichment). o Fold-change in gene expression of siARID1A upregulated genes, segregated based on ARID1A promoter-binding status ( $p=0.002$, unpaired Mann-Whitney $U$, two-tailed). Box-and-whiskers plotted in the style of Tukey without outliers. p mSigDb Hallmark pathway enrichment of $12 Z$ siARID1A differentially expressed genes (FDR < 0.0001). q Example browser tracks for ARID1A binding profile. Signal is displayed as log likelihood ratio (logLR). Single replicate signal is represented in light green, overlapping signal is represented in dark green. Green bars represent peaks called

expressed genes in siARID1A relative to control and siARID1A/ PIK3CA ${ }^{\text {H1047R }}$ relative to siARID1A (Fig. 6g). These 127 genes represent genes, which are differentially expressed by siARID1A, and further altered by the addition of PIK3CA ${ }^{\mathrm{H} 1047 \mathrm{R}}$. Of these genes, $47.2 \%$ were bound by ARID1A at the promoter in wild-type $12 \mathrm{Z}$ cells $\left(p<10^{-18}\right.$, hypergeometric enrichment) (Fig. 6h). We observed significant upregulation of these genes in siARID1A samples, and downregulation in siARID1A/ PIK3CA ${ }^{\text {H1047R }}$ (Fig. 6h, i). These genes were enriched for the hallmark EMT pathway, which was the most significant result 
a

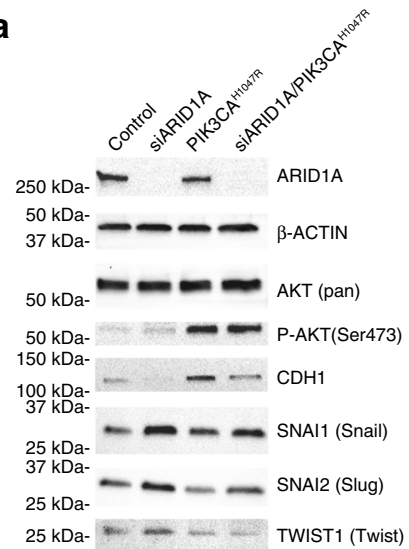

d

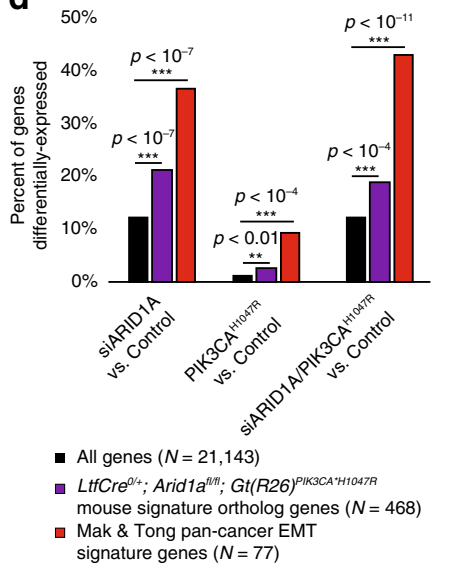

b

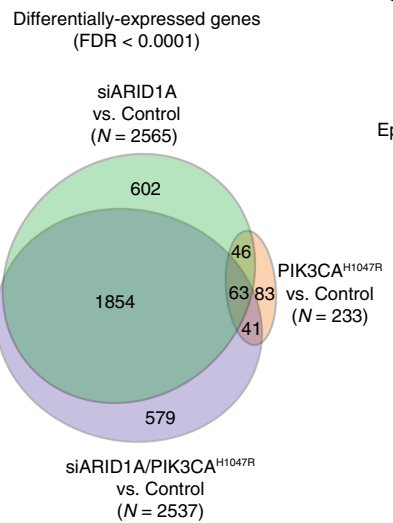

e Mak \& Tong pan-cancer EMT signature Upregulated in Downregulated in

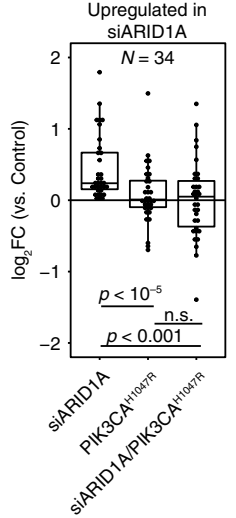

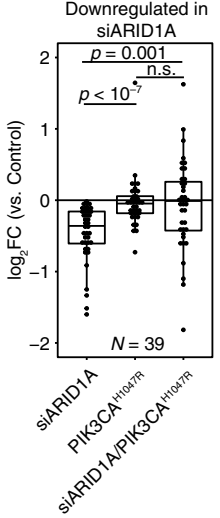

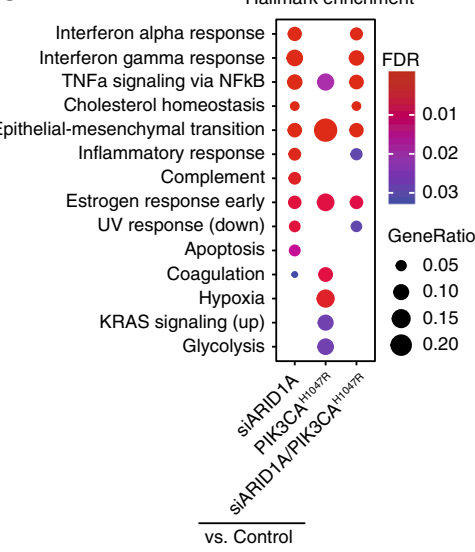

f

Hallmark EMT signature

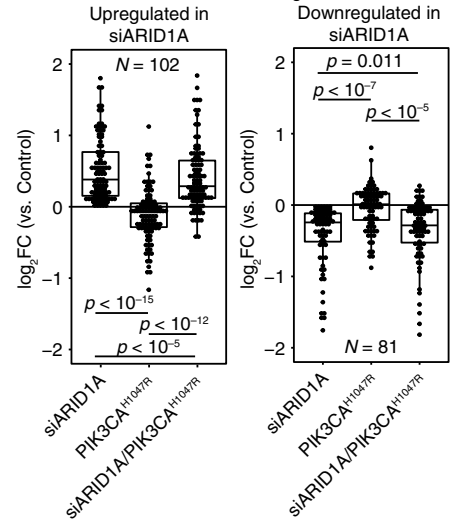

g

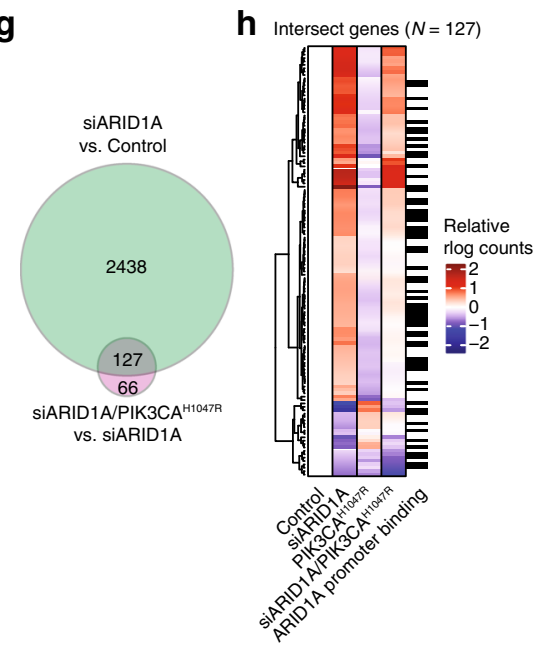

k

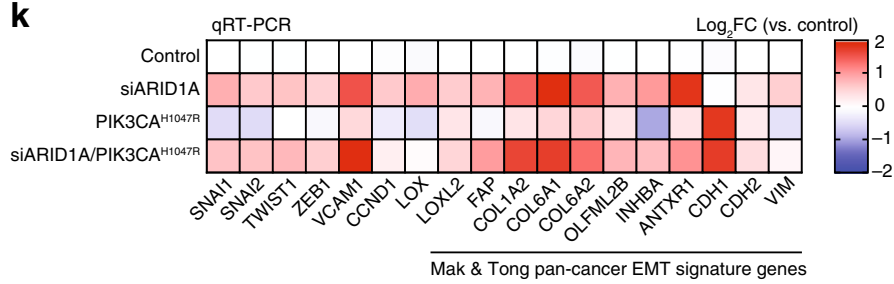

i Intersect genes $(N=127) \quad$ j
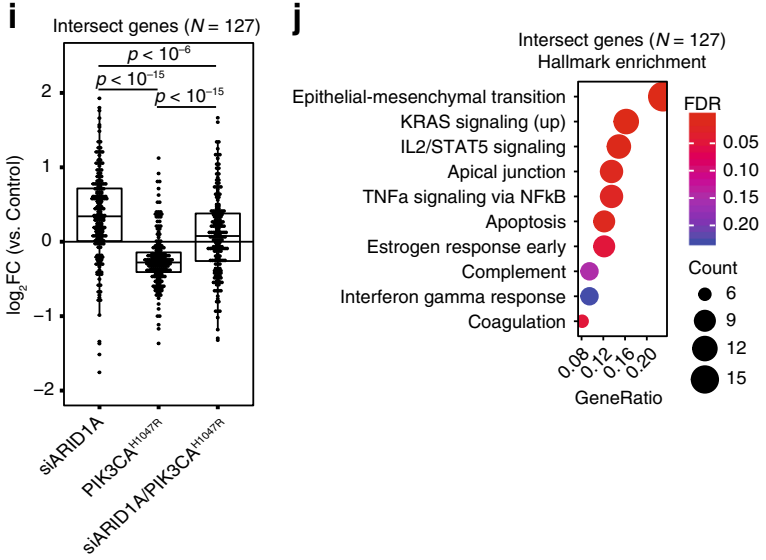

(Fig. 6j). The differential gene expression of EMT genes upon ARID1A loss was confirmed by quantitative reverse transcriptase (qRT)-PCR (Fig. 6k). These data provide further evidence that ARID1A loss induces a mesenchymal phenotype, which is antagonized by the PIK3CA ${ }^{\mathrm{H} 1047 \mathrm{R}}$ mutation, resulting in a partial EMT phenotype.
ARID1A loss and PIK3CA ${ }^{\mathrm{H} 1047 \mathrm{R}}$ promote invasive phenotypes. Partial EMT is associated with invasive phenotypes ${ }^{38}$, and EMT pathways play key roles in EC disease progression by promoting the invasion of epithelial cells into the myometrium ${ }^{44}$. To distinguish between the effect of ARID1A loss or PIK3CA ${ }^{\mathrm{H} 1047 \mathrm{R}}$ on invasive phenotypes, we co-transfected $12 \mathrm{Z}$ cells with a 
Fig. 6 PIK3CA ${ }^{\mathrm{H} 1047 \mathrm{R}}$ antagonizes ARID1A loss-induced mesenchymal phenotypes. a Western blot of ARID1A, $\beta$-Actin, AKT, P-AKT, CDH1, SNAI1, SNAI2, and TWIST1 following co-transfection of siNONtg and empty vector (control), siARID1A and empty vector (siARID1A), siNONtg and pPIK3CA ${ }^{\text {H1047R }}$ (PIK3CA H1047R), or siARID1A and pPIK3CA ${ }^{\mathrm{H} 1047 \mathrm{R}}$ (SIARID1A/PIK3CA H1047R). b Proportional Euler diagram displaying differentially expressed genes (FDR $<0.0001)$ from siARID1A, PIK3CA H1047R, and siARID1A/PIK3CA H1047R relative to control. c mSigDb Hallmark pathway enrichment for siARID1A,

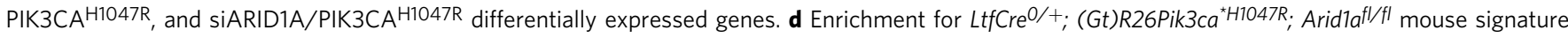
ortholog genes and Mak et al. pan-cancer gene signature within differentially expressed genes from siARID1A, PIK3CAH1047R, and siARID1A/PIK3CA H1047R relative to control. e, f Fold-change values of experimental groups relative to control for genes in the Mak and Tong pan-cancer EMT signature (e) and the Hallmark EMT signature (f), separated based on direction of gene expression change in siARID1A. Statistic represented is paired Mann-Whitney $U$ (twotailed). Box-and-whiskers plotted in the style of Tukey without outliers. $\mathbf{g}$ Intersection between siARID1A differentially expressed genes relative to control and siARID1A/PIK3CA ${ }^{H 1047 R}$ relative to siARID1A. $\mathbf{h}$ Heat map detailing relative expression of intersecting genes $(N=127)(F i g .6 \mathrm{~g})$ in control, siARID1A, PIK3CA ${ }^{\mathrm{H} 1047 \mathrm{R}}$, and siARID1A/PIK3CA ${ }^{\mathrm{H1047R}}$, and ARID1A promoter binding. These genes were enriched for ARID1A promoter binding ( $p<10^{-18}$, hypergeometric enrichment). i Expression level of intersect genes (Fig. 6g) in siARID1A, PIK3CA H1047R, and siARID1A/PIK3CA ${ }^{\mathrm{H} 1047 \mathrm{R}}$ relative to control. Statistic represented is paired Mann-Whitney $U$ (two-tailed). Box-and-whiskers plotted in the style of Tukey without outliers. $\mathbf{j}$ mSigDb Hallmark pathway enrichment for intersecting genes $(N=127)$ (Fig. 6g). $\mathbf{k}$ Changes in relative EMT gene expression upon ARID1A loss and PIK3CA H1047R overexpression as measured by qRT-PCR. Data represents three biological replicates

PIK3CA ${ }^{\text {H1047R }}$ expression plasmid and lentivirus expressing ARID1A short-hairpin RNAs (shRNAs) (shARID1A) (Fig. 7a). ARID1A knockdown induced migratory and invasive phenotypes in $12 \mathrm{Z}$ cells, and co-transfection with pPIK3CA ${ }^{\mathrm{H} 1047 \mathrm{R}}$ significantly enhanced migration and invasion (Fig. 7b, c). Cells treated with shARID1A displayed increased expression of F-actin (Fig. 7c). These results suggest that the co-mutation of ARID1A and PIK3CA in the endometrial epithelium promotes an invasive phenotype.

In vivo, we observed a requirement for both ARID1A loss and PI3K activation for invasive phenotypes. In $\mathrm{LtfCre}^{\mathrm{O} /+}$; $(\mathrm{Gt})$ R26Pik3ca ${ }^{*}$ H1047R; Aridlafl/t and LtfCre ${ }^{0 /+} ;$ (Gt)R26Pik3$c a^{*} H 1047 R$; Arid1 $a^{f l f l}$ mice, we observed invasion of endometrial epithelium into the myometrium (Fig. 7d). KRT8-positive epithelial cells migrated outside of the endometrium, invading a-smooth muscle actin ( $\alpha$-SMA)-positive myometrial cells and formed tumors (Fig. 7e). Invading epithelial cells contained a narrow leading edge and strand-like morphology, suggesting a collective migration of cells ${ }^{45}$. Some invasive sites formed welldifferentiated adenomas (Fig. 7d), while others were poorly differentiated clusters of tumor cells (Fig. 7d, e). Invasive KRT8positive epithelial glands were observed in direct contact with myometrial cells, often appearing as strands of epithelial cells trailing through the myometrial layers (Fig. 7d). These results suggest that ARID1A loss and PIK3CA ${ }^{H 1047 R}$ expression in the endometrial epithelium results in a partial EMT phenotype, promoting lesion formation and myometrial invasion (Fig. 7f).

\section{Discussion}

In this study, we found that ARID1A functions as a haploinsufficient tumor suppressor in the endometrial epithelium. $\mathrm{LtfCre}^{0 /+}$; (Gt)R26Pik3ca H1047R; Arid1afl/ + is sufficient to drive tumorigenesis and is nearly identical to $\mathrm{LtfCre} \mathrm{Cl}^{0 /+}$; $(\mathrm{Gt}) \mathrm{R} 26 \mathrm{Pik3-}$ $\mathrm{ca}^{*}{ }^{*} 1047 R$; Arid1 $a^{f l / f l}$ with respect to tumor burden, survival, gene expression, and chromatin accessibility changes. This is consistent with the spectrum of single-hit ARID1A mutations observed in EC, in which a majority of patients have only a single ARID1A nonsense mutation. Previous studies suggested ARID1A functions as a haploinsufficient tumor suppressor ${ }^{31}$ in ovarian $^{10,11}$, breast ${ }^{46}$, gastric ${ }^{47}$, and liver cancer ${ }^{48}$. ARID1A expression or mutation may not predict disease status, as singlehit mutations or epigenetic silencing may be sufficient for ARID1A-dependent changes in gene expression or transformation. Additionally, heterozygous loss of ARID1A may promote metastasis at late stages of the tumor progression, as observed in liver cancer ${ }^{49}$. ARID1A levels may be regulated throughout the menstrual cycle and mediate dissociation of decidua from the uterus. In this case, ARID1A heterozygosity may suffice for oncogenesis during points of low ARID1A expression, which may account for the ARID1A genetic differences observed between the present mouse model and epithelial ovarian cancer models ${ }^{29,30}$. This would explain the high ARID1A mutation rates in EC.

Previously, Raab et al. ${ }^{50}$ identified ARID1A binding preferentially at promoters in HepG2 liver cancer cells. In the present study, we show ARID1A enrichment at promoters, which was significantly correlated with chromatin accessibility. We observed increased accessibility at promoters upon ARID1A loss in human endometrial epithelial cells and, in vivo, in sorted mouse endometrial epithelial cells. Among direct ARID1A target genes, we also observed significant correlations between increasing promoter accessibility and increasing transcription of mesenchymal genes upon ARID1A loss. In addition, we observed greater activation of ARID1A target genes following ARID1A loss, as compared to ARID1A non-target genes. These data suggest ARID1Acontaining SWI/SNF complexes maintain endometrial epithelial cell identity by repressing genes required for transdifferentiation of epithelial cells into mesenchyme. ARID1A may promote endometrial plasticity by limiting the differentiation capacity of the epithelial cells. Repressive nucleosome positioning by ARID1A-containing SWI/SNF complexes may provide a barrier to transcriptional activation, as has been observed at the HIV $\mathrm{LTR}^{51}$

The data presented here demonstrate a cell-autonomous role for ARID1A in the preservation of endometrial epithelial cell identity and EMT regulation. In addition, we show $\mathrm{LtfCre} \mathrm{C}^{0 /+}$; $(\mathrm{Gt})$ R26Pik3ca ${ }^{*} H 1047 R$; Arid1aflffl cells gain VIM and ICAM-1 and invade the myometrium, but retain CDH1, EPCAM and KRT8 expression, suggesting an incomplete EMT phenotype ${ }^{38}$. VIM expression is upregulated in epithelial tumors of uterine corpus origin, but not epithelial tumors of ovarian origin ${ }^{52}$. ICAM-1 is expressed in migratory $\mathrm{EC}^{53}$, and is linked to increased peritoneal adhesion in endometriosis ${ }^{54}$. VIM and ICAM-1 may serve as markers of ARID1A-negative tumors of endometrial origin.

Partial or incomplete EMT is associated with invasive phenotypes in various cancers ${ }^{38,45}$. In EC, EMT is thought to play a role in myometrial invasion ${ }^{44}$. In this study, we found that ARID1A loss and PI3K activation in endometrial epithelium leads to enhanced migration and invasion in vitro and myometrial invasion in vivo, reflecting the myometrial invasion phenotypes observed clinically. Myometrial invasion in EC correlates with distal metastases, disease recurrence, and adenomyosis ${ }^{55-57}$. EC patients with gene expression signatures most similar to $\mathrm{LtfCre} \mathrm{C}^{\mathrm{O}++}$; (Gt)R26Pik3ca ${ }^{{ }^{*} H 1047 R}$; Arid1aflfl had greater tumor invasion and higher tumor grade. The collective migration of mutant endometrial epithelium undergoing partial EMT may enhance the invasive properties of EC, permitting myometrial invasion. 
a

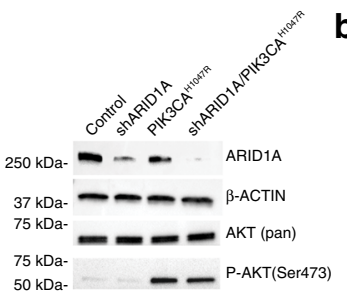

b
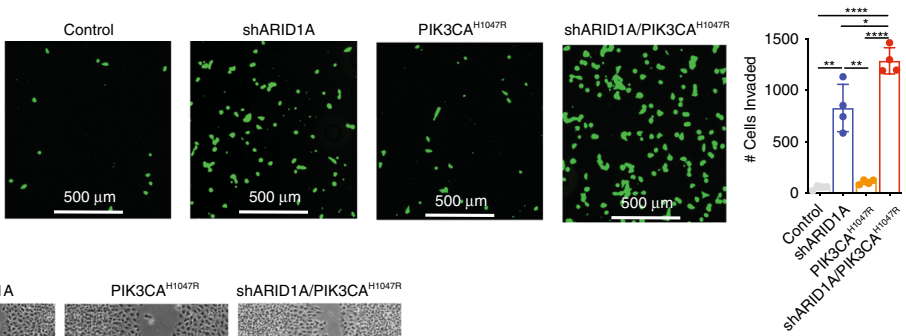
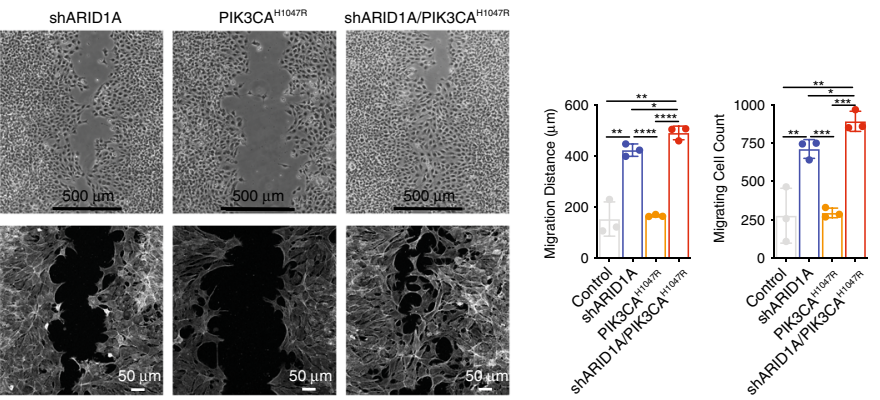

d
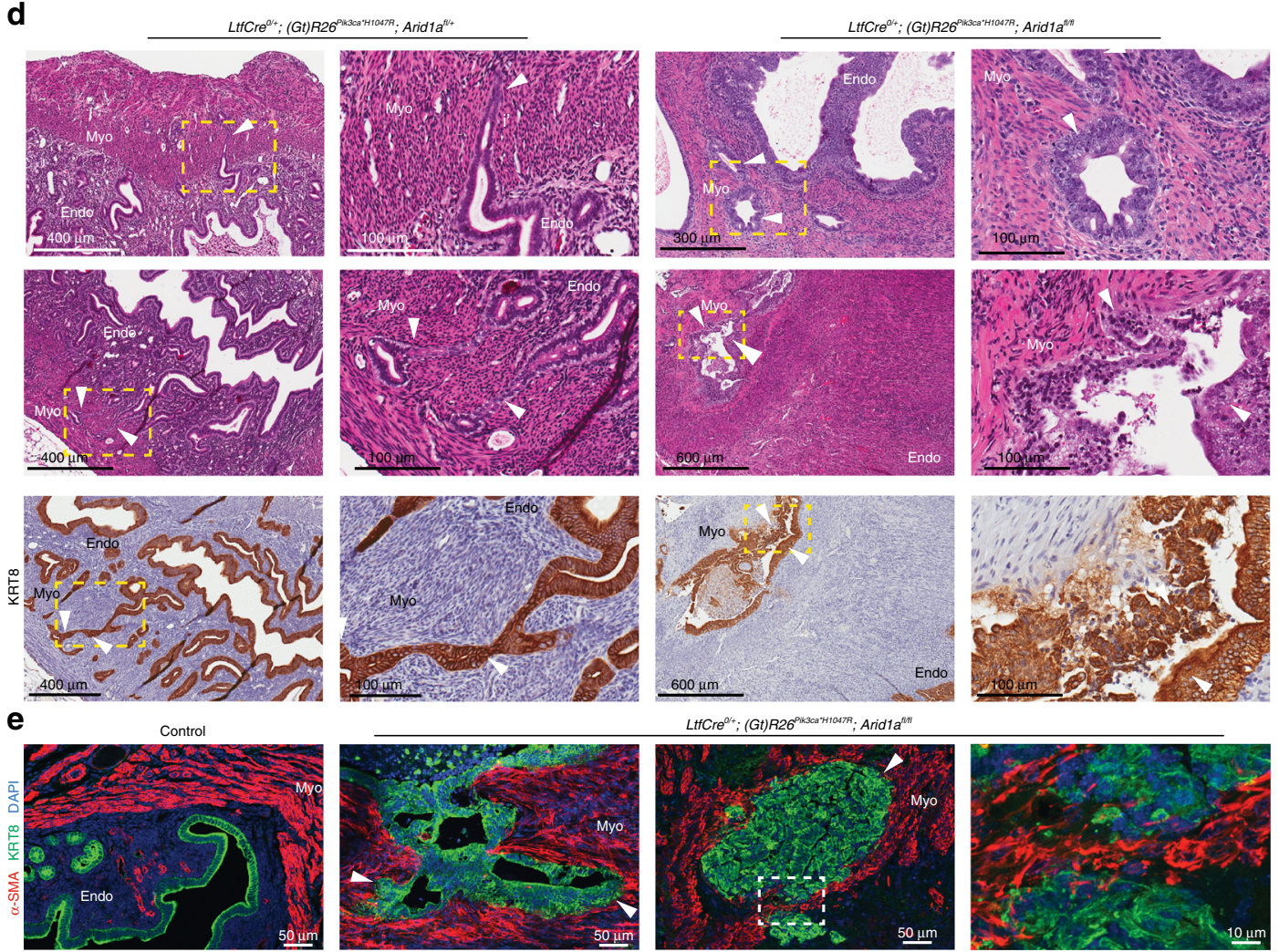

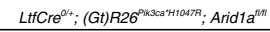
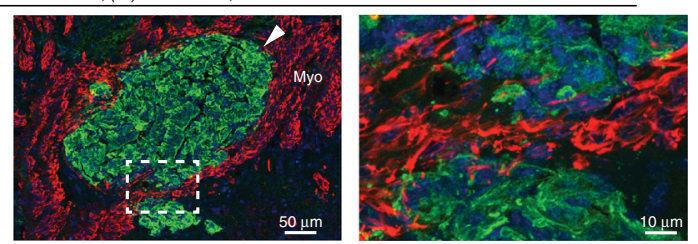

f

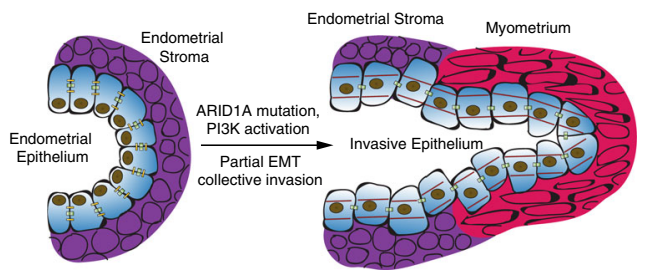

The retention of some epithelial characteristics upon PIK3$\mathrm{CA}^{\mathrm{H} 1047 \mathrm{R}}$ expression may facilitate the establishment of epithelial tumors ${ }^{58}$. Epithelial transdifferentiation is a proposed mechanism by which normal epithelia convert into abnormal epithelia without undergoing an mesenchymal cell intermediate ${ }^{59}$. PIK3CA mutation is an early event in atypical hyperplasia ${ }^{24}$,

whereas loss of ARID1A immunoreactivity correlates with malignant transformation in endometrial cancer ${ }^{15}$. A recent study identified PIK3CA as being commonly mutated in endometrial glands, often without transformation, suggesting PIK3CA mutation as an early event, with ARID1A mutation coming later in the progression of endometriosis ${ }^{17}$. ARID1A mutations have 
Fig. 7 ARID1A loss and PIK3CA H1047R promote myometrial invasion in vivo and migration in vitro. a Western blot of ARID1A, $\beta$-Actin, AKT, P-AKT, following co-transfection of shNONtg and empty vector (control), shARID1A and empty vector (shARID1A), shNONtg and pPIK3CA H1047R (PIK3CA ${ }^{\text {H1047R) }}$ or shARID1A and pPIK3CA H1047R (shARID1A/PIK3CA H1047R). b Invasion assay of $12 Z$ cells with ARID1A loss and PIK3CA H1047R overexpression. Representative images of calcein AM-stained cells are and total invaded cell counts are shown (scale bar $=500 \mu \mathrm{m}$ ). Data represents four biological replicates (mean \pm s.d; ${ }^{\star} p<0.05,{ }^{\star \star} p<0.01,{ }^{\star \star \star \star} p<0.0001$, unpaired $t$-test, two-tailed). c Migration assay of $12 Z$ cells with ARID1A loss and PIK3CA H1047R overexpression. Upper images are representative of cells $24 \mathrm{~h}$ following removal of insert (scale bar $=500 \mu \mathrm{m}$ ). Lower images are maximum intensity confocal projections of cells stained with fluorescent phalloidin to label with F-actin (scale bar $=50 \mu \mathrm{m}$ ). Average Migration represents the average difference distance across each migration front from 0 to $24 \mathrm{~h}$. Migrating cell counts represent number of cells in migration area after $24 \mathrm{~h}$. Data represents three biological replicates (mean $\pm \mathrm{s} . \mathrm{d} ;{ }^{\star} p<0.05,{ }^{\star \star} p<0.01,{ }^{\star \star \star} p<0.001,{ }^{\star \star \star \star *} p<0.0001$, unpaired $t$-test, two-tailed). d Myometrial

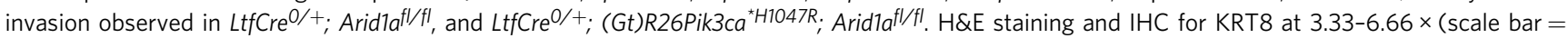
$300-600 \mu \mathrm{m}$, as stated on figure) and $\times 20$ (scale bar $=100 \mu \mathrm{m}$ ) magnification, with $\times 20$ magnifications representing portion panel to the right surrounded by yellow box. White arrows indicate invasive endometrial epithelium. Endo, endometrium; Myo, myometrium. e Images of maximum intensity confocal projections of control and $\mathrm{LtfCre} /++;(G t) R 26 P i k 3 c^{*}{ }^{*} 1047 R$; Aridlafl/fl endometrium sections stained with $\alpha$-smooth muscle actin ( $\alpha$-SMA) (red), KRT8 (green) and counter-stained with DAPI (blue) $(N \geq 3$ ). White arrows indicate invasive endometrial epithelium (scale bar $=50$ or $10 \mu \mathrm{m}$, as stated on figure). $\mathbf{f}$ Diagram representation of EMT-induced invasive endometrial epithelium following ARID1A loss and PIK3CA $\mathrm{H1047R}$ mutation

previously been implicated in invasion during metastasis ${ }^{49,60-62}$. In the LtfCre ${ }^{0 /+}$; (Gt)R26Pik3ca ${ }^{*} H 1047 R$; Arid1afl/fl endometrial epithelium, PI3K activation may partially suppress the full acquisition of mesenchymal phenotypes upon ARID1A loss, resulting in an abnormal epithelial state with invasive properties. PI3K activation may also allow cells to bypass the endometrial epithelial cell apoptosis observed in $\mathrm{LtfCre} \mathrm{C}^{0 /+}$; Arid1aflfl mice. This may be another reason why ARID1A mutations are commonly observed alongside activating PI3K mutations in neoplasms originating from the endometrial epithelium.

The partial EMT phenotype may increase the invasive potential of the endometrium. The expression of EMT factors is increased at the myoinvasive front of $\mathrm{ECs}^{44}$, suggesting collective migration rather than single cell metastasis ${ }^{63}$. Endometriotic lesions retain $\mathrm{CDH} 1$ expression $^{64}$, suggesting collective migration rather than metastasis via a single cell ${ }^{63}$. Within primary tumors, adjacent cells may differentiate into different intermediate stages along the EMT-spectrum due to differing stimulus within the tumor microenvironment, including surrounding stroma ${ }^{38}$. Invasive, mesenchymal-like cells may lead the way for cohorts of epithelial cells with which they retain some cell-cell junctions ${ }^{63}$. Upon arrival to metastatic sites, lack of stromal signals present at the site of origin may allow for epithelial gland formation ${ }^{58}$. This may explain the formation of endometrial glands outside of the endometrium derived from cells with mesenchymal-like invasiveness.

EC survival rates are high if the disease is detected at an early stage when the tumors are still confined to the endometrium. Myometrial invasion or tumor dissemination to other sites in the body correlates with poor survival. The notion that collective epithelial invasion promotes EC metastasis may lead to therapeutic options for patients with disseminated disease. The identification of pathways involved in the collective invasion may lead to the development of anti-metastatic drugs.

\footnotetext{
Methods

Mice. All mice were maintained on an outbred genetic background using CD-1 mice (Charles River). (Gt)R26Pik3ca ${ }^{*} H 1047 R$ and LtfCre (Tg(Ltf-iCre) 14Mmul) alleles were purchased from The Jackson Laboratory and identified by PCR using published methods ${ }^{32,33}$. Arid1afl and Arid1a ${ }^{V 1068 G}$ alleles were distinguished by $\mathrm{PCR}^{20,30}$. For detection of Arid $1 a^{V 1068 G}$ allele, PCR product was treated with HincII at $37^{\circ} \mathrm{C}$ for $1 \mathrm{~h}$. Genotyping primers are listed in Supplementary Table 1 . Uncropped genotyping gels can be found in Supplementary Fig. 7. Endpoints were vaginal bleeding, severe abdominal distension, and signs of severe illness, such as dehydration, hunching, jaundice, ruffled fur, signs of infection, or nonresponsiveness. Sample sizes within each genotype were chosen based on the proportions of animals with vaginal bleeding between each experimental group or a Kaplan-Meyer log-rank test for survival differences. For weight measurements,
} uteri were collected at time of sacrifice and placed immediately into neutral- buffered formalin at $4{ }^{\circ} \mathrm{C}$. After $24 \mathrm{~h}$, tissues were washed with phosphate-buffered saline (PBS) and $50 \% \mathrm{EtOH}$, placed in $70 \% \mathrm{EtOH}$, and then weighed. Mice were housed at the Van Andel Research Institute Animal Facility and the Michigan State University Grand Rapids Research Center in accordance with protocols approved by Michigan State University.

Cell lines. $12 Z$ immortalized human endometrial epithelial cells were provided by the laboratory of Asgi Fazleabas ${ }^{40}$. $12 \mathrm{Z}$ cells were maintained in Dulbecco's Modified Eagle Media (DMEM)/F12 media supplemented with 10\% fetal bovine serum (FBS), $1 \%$ L-glutamine and 1\% penicillin/streptomycin (P/S). Lenti- $\mathrm{X}^{\mathrm{TM}}$ 293T (Clontech, Cat\# 632180, CVCL_0063) cells were maintained in DMEM + $110 \mathrm{mg} / \mathrm{L}$ Sodium Pyruvate (Gibco) supplemented with $10 \%$ FBS, 1\% L-glutamine, $1 \%$ P/S. Cell line validation for the $12 Z$ cell line was performed by IDEXX BioResearch: the $12 \mathrm{Z}$ cell line has a unique profile not found in the current public databases. The $12 \mathrm{Z}$ and Lenti-X 293T cell lines tested negative for mycoplasma contamination. Testing was performed using the Mycoplasma PCR Detection Kit (Applied Biological Materials). No commonly misidentified cell lines were used in this study.

Histology and immunohistochemistry. For indirect immunohistochemistry (IHC), 10\% neutral-buffered formalin (NBF)-fixed paraffin sections were processed for heat-based antigen unmasking in $10 \mathrm{mM}$ sodium citrate [pH 6.0]. Sections were incubated with antibodies at the following dilutions: 1:200 ARID1A (D2A8U) (12354, Cell Signaling); 1:400 Phospho-S6 (4585, Cell Signaling); 1:100 KRT8 (TROMA1, DHSB); 1:100 EPCAM (G8.8-s, DHSB); 1:400 PGR (SAB5500165, Sigma). TROMA-I antibody was deposited to the DSHB by Brulet, P./Kemler, R. (DSHB Hybridoma Product TROMA-I). EPCAM antibody (G8.8) was deposited to the DSHB by Farr, A.G. (DSHB Hybridoma Product G8.8). Antibody details are listed in Supplementary Table 2. The following Biotin-conjugated secondary antibodies were used: donkey anti-rabbit IgG (711-065-152, Jackson Immunoresearch Lab) and donkey anti-rat IgG (\#705-065-153, Jackson Immuno-research Lab). Secondary antibodies were detected using VECTASTAIN Elite ABC HRP Kit (Vector). Sections for IHC were lightly counter-stained with Hematoxylin QS or Methyl Green (Vector Labs). Routine Hematoxylin and Eosin (H\&E) staining of sections was performed by the Van Andel Research Institute (VARI) Histology and Pathology Core. A VARI animal pathologist reviewed histological tumor assessments.

Immunofluorescence. For indirect immunofluorescence, tissues were fixed in $4 \%$ paraformaldehyde. Frozen samples were sectioned at $10 \mu \mathrm{m}$ on a CM3050 S cryostat (Leica) and collected on white frosted, positive charged ultra-clear microscope slides (Denville). Frozen slides were post-fixed with 2\% PFA/1 PBS, and permeabilized with $0.3 \%$ TX100 in PBS, and treated with $100 \mathrm{mM}$ glycine/1x PBS [pH 7.3]. Primary antibodies were applied to slides at the following dilutions: 1:200 ARID1A (D2A8U) (12354, Cell Signaling); 1:100 KRT8 (TROMA1, DHSB); 1:100 EPCAM (G8.8-s, DHSB); 1:50 ZO-1 (61-7300, ThermoFisher); 1:200 CDH1 (3195, Cell Signaling); 1:100 CLDN10 (38-8400, ThermoFisher); 1:100 VIM (5741, Cell Signaling); 1:400 PGR (SAB5500165, Sigma); 1:200 ERa (ab32063, abcam); 1:2000 SMA (Sigma, C618); 1:100 SNAI2 (9585, Cell Signaling); 1:40 ICAM-1 (AF796-SP, R\&D Systems). Secondary antibodies used were: 1:500 donkey antirabbit IgG, alexa fluor 555-conjugated antibody (\#A-31572, ThermoFisher); 1:500 goat anti-rabbit IgG, alexa fluor 555-conjugated antibody (\#A-21428, ThermoFisher); 1:500 goat anti-rat IgG, alexa fluor 647-conjugated antibody (A-21247, ThermoFisher); 1:250 donkey anti-rat IgG, alexa fluor 647-conjugated antibody (712-605-153, Jackson Immuno-Research Lab); 1:250 donkey anti-goat fluor 488conjugated antibody (705-545-147, Jackson Immuno-Research Lab). PhalloidiniFluor 594 (1:1000, abcam) was used to stain F-actin. Auto-fluorescence was 
quenched using the TrueVIEW Auto-fluorescence Quenching Kit (Vector Laboratories). ProLong Gold Antifade Reagent with DAPI (8961, Cell Signaling) was used for DAPI staining.

Microscopy and imaging. Confocal images were taken on a Nikon Eclipse Ti inverted microscope using a Nikon $\mathrm{C} 2$ + confocal microscope laser scanner. Confocal immunofluorescent images are representative maximum intensity projections.

Cell sorting. Mouse uteri were surgically removed and minced using scissors. Tissues were digested using the MACS Multi Tissue Dissociation Kit II (Miltenyi Biotec) for $80 \mathrm{~min}$ at $37^{\circ} \mathrm{C}$. Digested tissues were strained through a $40 \mu \mathrm{m}$ nylon mesh (ThermoFisher). The Red Cell Lysis Buffer (Miltenyi Biotec) was used to remove red blood cells. Dead cells removed using the MACS Dead Cell Removal Kit (Miltenyi Biotec), and EPCAM-positive cells were positively selected and purified using a PE-conjugated EPCAM antibody and anti-PE MicroBeads (Miltenyi Biotec), per the manufacturers' instructions. A BD Accuri C6 flow cytometer (BD Biosciences) was used to confirm purity of EPCAM-positive population.

RNA isolation and qRT-PCR. The Arcturus PicoPure RNA Isolation Kit (ThemoFisher), including an on-column DNA digestion using the RNAse-free DNAse set (Qiagen), was used to purify RNA from in vivo EPCAM-sorted endometrial epithelial cells. To confirm loss of ARID1A transcript in EPCAM-positive $\mathrm{LtfCre}^{\mathrm{O}++}$; (Gt)R26Pik3ca ${ }^{* H 1047 R}$; Arid1aflfl cells, complementary DNA (cDNA) was synthesized from RNA, and qRT-PCR was performed using Ssofast PCR master mix (Biorad) using previously described primers ${ }^{20}$ and the Applied Biosystems ViiA7 real-time PCR system. ARID1A expression was normalized to GAPDH. For in vitro experiments, RNA samples were collected $72 \mathrm{~h}$ post siRNA transfection using the Quick-RNA Miniprep Kit (Zymo Research). cDNA was synthesized from RNA, and qRT-PCR was performed using PowerUp SYBR Green Master Mix (ThermoFisher) and the Applied Biosystems ViiA7 real-time PCR system. Primer pairs for human genes are described in Supplementary Table 1.

RNA-seq. Libraries were prepared by the Van Andel Genomics Core from $100 \mathrm{ng}$ of total RNA for mouse samples, and Lexogen SIRV-set2 RNAs (Lexogen GmbH, Vienna Austria) were spiked into RNA prior to library preparation at a concentration of $1 \%$ by mass. For human samples, $500 \mathrm{ng}$ of total RNA material was used as input, with no spike in. For all samples, libraries were generated using the KAPA Stranded mRNA-Seq Kit (v4.16) (Kapa Biosystems, Wilmington, MA USA). RNA was sheared to 250-300 bp and reverse transcribed. Prior to PCR amplification, cDNA fragments were ligated to Bio Scientific NEXTflex Adapters (Bio Scientific, Austin, TX, USA). Quality and quantity of the finished libraries were assessed using a combination of Agilent DNA High Sensitivity chip (Agilent Technologies, Inc.), QuantiFluor dsDNA System (Promega Corp., Madison, WI, USA), and Kapa Illumina Library Quantification qPCR assays (Kapa Biosystems). All libraries were pooled equimolarly, and single end sequencing to a minimum depth of $30 \mathrm{M}$ reads per library was performed using an Illumina NextSeq 500 sequencer using a 75 bp sequencing kit (v2) (Illumina Inc., San Diego, CA, USA). Base calling was done by Illumina NextSeq Control Software (NCS) v2.0 and output of NCS was demultiplexed and converted to FastQ format with Illumina Bcl2fastq v1.9.0.

RNA-seq analysis. Raw 75 bp reads were trimmed with cutadapt $t^{65}$ and Trim Galore! (http://www.bioinformatics.babraham.ac.uk/projects/trim_galore/) followed by quality control analysis via Fast $\mathrm{C}^{66}$. Trimmed mouse reads were aligned to $\mathrm{mm} 10$ genome assembly and indexed to GENCODE ${ }^{67}$ vM16 GFF3 annotation via $S T A R^{68}$ aligner with flag '-quantMode GeneCounts' for feature counting, and human reads were aligned to GRCh38.p12 and indexed to GENCODE v28. For mouse libraries, Lexogen SIRVome was independently aligned and quantified for qualitative assessment of library concordance. Output gene count files were constructed into an experimental read count matrix in R. Low count genes were filtered ( 1 count per sample on average) prior to DESeq $2^{69,70}$ count normalization and subsequent differential expression analysis. Calculated differential expression probabilities were corrected for multiple testing by independent hypothesis weighting $(\mathrm{IHW})^{71}$ for downstream analysis. Differentially expressed gene thresholds were set at FDR $<0.05$ for mouse data and FDR $<0.0001$ for human data. All reported instances of $\log _{2}$ (fold-change) data from RNA-seq are adjusted by DESeq 2 original shrinkage estimator except for TCGA-UCEC comparisons and statistical comparisons between $\log _{2}(\mathrm{FC})$ values, which use non-adjusted values. Principal component analysis was calculated using DESeq2 from top 500 genes by variance across samples. RNA-seq heatmaps were generated using scaled regularized-logarithm ( $\mathrm{rlog}$ ) counts for visualization, or relative to controls by subtracting mean rlog counts. LtfCre ${ }^{0 /+} ;(G t) R 26^{P i k 3 c a^{*} H 1047 R}$; Aridla ${ }^{f l / f l}$ signature genes were defined by $\mathrm{FDR}<10^{-5}$ and $\left|\log _{2}(\mathrm{FC})\right|>1$.

ATAC-seq. Libraries were prepared following previously described methods ${ }^{39,72,73}$ Mouse endometrial cells were isolated using methods described above. For purified mouse endometrial epithelium and $12 \mathrm{Z}$ cells, between 25,000 and 50,000 cells were resuspended in cold lysis buffer (10 mM Tris-HCL [pH 7.4], $10 \mathrm{mM} \mathrm{NaCl}, 3 \mathrm{mM}$ $\left.\mathrm{MgCl}_{2}, 0.1 \% \mathrm{NP}-40\right)$ and centrifuged at $500 \times g, 4{ }^{\circ} \mathrm{C}$ for $10 \mathrm{~min}$ to isolate nuclei. Nuclei were treated with Tn5 Transposase for $30 \mathrm{~min}$ at $37^{\circ} \mathrm{C}$ using the Nextera DNA Library Prep Kit (Illumina). DNA was isolated using the Qiagen MinElute Reaction Cleanup Kit. Libraries were amplified using barcoded primers for 1-8 cycles as described ${ }^{39}$. Libraries were purified using Kapa Pure Beads to remove primer dimers and $>1000$ bp fragments. Libraries were sequenced by the Van Andel Genomics Core. Quality and quantity of the finished libraries were assessed using a combination of Agilent DNA High Sensitivity chip (Agilent Technologies, Inc.), QuantiFluor dsDNA System (Promega Corp., Madison, WI, USA), and Kapa Illumina Library Quantification qPCR assays (Kapa Biosystems). All libraries were pooled equimolarly, and paired end sequencing to a minimum depth of $20 \mathrm{M}$ reads per library was performed using an Illumina NextSeq 500 sequencer using a $150 \mathrm{bp}$ sequencing kit (v2) (Illumina Inc., San Diego, CA, USA). Base calling was done by Illumina NextSeq Control Software (NCS) v2.0 and output of NCS was demultiplexed and converted to FastQ format with Illumina Bcl2fastq v1.9.0.

ATAC-seq analysis. Libraries were combined across flow cells and trimmed with cutadapt and Trim Galore! followed by quality control analysis via FastQC. Trimmed reads were aligned to $\mathrm{mm} 10$ mouse reference genome via Bowtie $2^{74}$ with flags '-very-sensitive' and '-X 1000' in concordance with the library size-selection step, and, similarly, human reads were aligned to GRCh38.p12 using the same parameters ${ }^{75}$. Reads were then sorted and indexed with samtools ${ }^{76}$. Mitochondrial reads were then discarded from BAMs, using Harvard ATAC-seq module removeChrom script (https:/github.com/harvardinformatics/ATAC-seq), and subsequently filtered for only properly paired reads by samtools view $-f 3$. At this step, working library complexity was estimated by ATACseqQC::

estimateLibComplexity 77,78 . To compensate for differing library complexities within an experimental design, we normalized by randomly subsampling libraries to a calculated fraction of the original library, as estimated by the bootstrap interpolation, via samtools view with flag '-s' to achieve normalized library sizes. After subsampling libraries to lowest complexity, PCR duplicates were removed with Picard MarkDuplicates (http://broadinstitute.github.io/picard/), and reads were finally name-sorted prior to conversion to BEDPE format with bedtools ${ }^{79}$ bamtobed with flag '-bedpe'. BEDPE coordinates were then shifted 4 and 5 bp to correct for TN5 transposase integration ${ }^{39}$, and the standard BEDPE files were rewritten to a minimal BEDPE format, as defined by MACS2 manual, through an awk script. MACS2 ${ }^{80}$ was used to call broad peaks from final minimal BEDPE fragment coordinates with FDR $<0.05$ threshold and no control input, and the resulting peaks were repeat-masked by blacklist filtering ${ }^{81}$. A naive overlap peak set, as defined by ENCODE, was constructed for each biological condition by combining replicates and calling broad peaks on pooled BEDPE files followed by intersectBed to select for peaks of at least $50 \%$ overlap with each biological replicate

Differential accessibility was calculated by firstly defining a more relaxed consensus peak set $p=\left(\bigcap_{j=1}^{n} e_{j}\right) \cup\left(\bigcap_{j=1}^{n} c_{j}\right)$ for any partial intersect where $e_{1}, \ldots, e_{\mathrm{n}}$ are MACS2 peak sets from biological replicates of the experimental condition, and $c_{1}$, $\ldots, c_{n}$ are peak sets from control biological replicates. This consensus peak set was used in $c s a w^{82}$ as coordinates for counting reads within specified windows, with additional parameters set to restrict windows to standard chromosomes and nonblacklisted regions. Windows $>1$ kilobase in width were filtered along with low read-abundance windows $(\log \mathrm{CPM}<-3)$. In order to compensate for differing efficiencies of reactions between libraries, a non-linear loess-based normalization approach was employed to remove trended biases. This method was empirically determined to elicit the most conservative results as opposed to other approaches to window count normalization. csaw uses $e d g e R^{83}$ quasi-likelihood functionality to calculate differential accessibility, for which FDR thresholds were used to determine final differential peak sets (FDR $<0.20$ mouse data; FDR $<0.05$ human data). Finally, proximal windows within $500 \mathrm{bp}$ were merged, and the most significant window statistic was used to represent the merged window.

Significant differentially accessible genomic regions were annotated by $H O M E R^{84}$ with a modification to cis-promoter classification as within $3000 \mathrm{bp}$ of a canonical gene TSS, which remains consistent throughout all reported analyses. HOMER de novo motif enrichment and genome ontology was performed on all significant differentially accessible genomic regions. Common differential mouse ATAC/RNA genes were selected by the presence of a differentially accessible promoter ATAC peak (FDR $<0.20)$ and RNA-seq differential expression $(F D R<$ $0.05)$.

Analysis of TCGA-UCEC data. ARID1A alteration incidence analysis was calculated using the TCGA Pan-Can UCEC 22 cohort $(N=509)$ retrieved from cBioPortal $^{85}$. All molecular data for subsequent analyses was pulled from the 28th January, 2016 release of Broad GDAC Firehose (https://doi.org/10.7908/ C11G0KM9). For molecular comparisons, patients were considered ARID1A ${ }^{\text {mut }}$ if they had somatic alterations (excluding missense and synonymous mutations) and ARID1A $^{\text {wt }}$ if no alterations were detected at the ARID1A locus. RNASeqV2 $\mathrm{RSEM}^{86}$ normalized gene counts were quantile normalized prior to filtering lowcount genes (one count per sample on average) and fitting linear models via limma ${ }^{87}$ for differential expression analysis in subsets of patients. Moderated statistics were calculated by empirical Bayes moderation via limma::eBayes with 
arguments 'trend $=$ TRUE' and 'robust $=$ TRUE', and probabilities were adjusted for multiple testing by FDR. Additional metrics for clinical staging and tumor invasion were acquired from the GDC ${ }^{88}$ TCGA-UCEC dataset $(N=605)$ in UCSC $\mathrm{Xena}^{28}$. Broad GSEA ${ }^{89}$ for mSigDb v6.2 Hallmark pathways ${ }^{90}$ was performed on ortholog-converted DESeq2 normalized counts from generated mouse data and RNASeqV2 RSEM normalized counts from TCGA-UCEC data. Broad $s S G S E A^{91}$ was also performed on RNASeqV2 RSEM normalized counts from TCGA-UCEC data. Orthologs of the mouse gene signature established herein were used to define UCEC endometrioid patients in ssGSEA-enriched or unenriched quartiles, which reflect mouse model transcriptome.

Bioinformatics and statistics. The 77 gene Pan-Cancer EMT signature was extracted from Supplementary Table S2 of Mak et al. ${ }^{37}$. Various ClusterProfiler ${ }^{92}$ functions were used to calculate and visualize pathway enrichment from a list of gene symbols or Entrez ${ }^{93}$ IDs with respective gene universes. biomaR $t^{94,95}$ was used for all gene nomenclature and ortholog conversions. ggplot $2^{96}$ was used for various plotting applications. ComplexHeatmap ${ }^{97}$ was used for hierarchical clustering by Euclidean distance and visualization. eulerr was used to produce proportional Euler diagrams $s^{98}$. The cumulative hypergeometric distribution was used for enrichment tests performed throughout this manuscript. The statistical computing language $\mathrm{R}$ was used for many applications throughout this manuscript ${ }^{99}$. HOMER was used to compute integer read counts at loci of interest for tag density heatmaps and scatter plots. TxDb.Hsapiens.UCSC.hg38.knownGene was used to generate promoter regions for all standard hg38 genes ${ }^{100}$.

Transfection of $12 Z$ cells with siRNA and plasmid DNA. $12 Z$ cells were seeded at a density of 40,000 cells $/ \mathrm{mL}$ in DMEM/F12 media supplemented with $10 \%$ FBS and $1 \%$ L-glutamine. The following day, cells were transfected with $50 \mathrm{pmol} / \mathrm{mL}$ of siRNA (Dharmacon, ON-TARGETplus Non-targeting Pool and human ARID1A \#8289 SMARTpool) using the RNAiMax (ThermoFisher) lipofectamine reagent according to the manufacturer's instructions at a ratio of 1:1 volume:volume in OptiMEM (Gibco). After $24 \mathrm{~h}$, the media was replaced. ATAC samples were collected after $48 \mathrm{~h}$. For plasmid co-transfection experiments, $24 \mathrm{~h}$ after siRNA transfection, cells were transfected with 500 ng pBabe vector containing PIK3$\mathrm{CA}^{\mathrm{H} 1047 \mathrm{R}}$ (pPIK3CA $\left.{ }^{\mathrm{H} 1047 \mathrm{R}}\right)$ or pBabe empty vector using the FuGene HD transfection reagent (Promega) according to the manufacturers' instructions at a ratio of 2:1 volume:mass, and media was replaced after $4 \mathrm{~h}$. The PPIK3CA $\mathrm{CH}^{\mathrm{H}} 047 \mathrm{R}$ was a gift from Jean Zhao (Addgene plasmid 12524) ${ }^{101}$. The following day, media was replaced with DMEM/F12 media supplemented with $0.5 \%$ FBS, $1 \% \mathrm{P} / \mathrm{S}$, and $1 \% \mathrm{~L}$-glutamine. Cells were collected $72 \mathrm{~h}$ post siRNA transfection using the QuickRNA Miniprep Kit (Zymo Research) for RNA or RIPA buffer (Cell Signaling) for protein.

Generation of lentiviral shRNA particles. Lentiviral particles expressing shRNAs were produced in $293 \mathrm{~T}$ cells according to the manufacturers' instructions. Briefly, Lenti-X ${ }^{\mathrm{TM}} 293 \mathrm{~T}$ cells were transfected with lentiviral packaging mix (Sigma) and MISSION pKLO.1 plasmid containing non-targeting shRNA (shNONtg) or pooled ARID1A shRNAs (shARID1A) (Sigma) using polyethylenimine (PEI) in DMEM $+4.5 \mathrm{~g} / \mathrm{L}$ D-Glucose, $110 \mathrm{mg} / \mathrm{L}$ Sodium Pyruvate, $10 \% \mathrm{FBS}, 1 \% \mathrm{~L}$-glutamine. After $4 \mathrm{~h}$, media was replaced with DMEM/F12, 10\% FBS, $1 \%$ L-glutamine, $1 \% \mathrm{P} / \mathrm{S}$. Viral particles were collected after 48 and $96 \mathrm{~h}$, and viral titers were calculated using the qPCR Lentiviral Titration Kit (ABM).

Migration assay. $12 \mathrm{Z}$ cells were seeded into $35 \mathrm{~mm}$ dishes containing four-well culture inserts at a density of 4000 cells per well. After $24 \mathrm{~h}$, cells were transfected with $125 \mathrm{ng}$ pBabe vector or pPIK3CA ${ }^{\mathrm{H} 1047 \mathrm{R}}$ using the FuGene HD as described above. After $4 \mathrm{~h}$, cells were treated with lentiviral particles expressing shNONtg or shARID1A at a multiplicity of infection of 100 . After $24 \mathrm{~h}$, the media was replaced. At $48 \mathrm{~h}$ post transfection, media was replaced with serum-free DMEM/F12 containing $1 \% \mathrm{~L}$-glutamine and $1 \% \mathrm{P} / \mathrm{S}$. After $16 \mathrm{~h}$ of serum deprivation, culture inserts were removed and serum-free media was added. At 0 and $24 \mathrm{~h}$, images were taken using a Nikon Eclipse Ti microscope. Distances between migration fronts were measured using NIS Elements Advanced Research software at 16 different points $100 \mu \mathrm{m}$ apart. Migration distance was calculated by subtracting the average distance across migration fronts at $24 \mathrm{~h}$ from the average distance at $0 \mathrm{~h}$. Cells counts were conducted within a $1500 \mu \mathrm{m}$ by $700 \mu \mathrm{m}$ window surrounding the migration area.

Invasion assay. $12 \mathrm{Z}$ cells were seeded in six-well dishes at a density of 50,000 cells per well. After $24 \mathrm{~h}$, cells were transfected with pPIK3CA or empty vector as described above. After $4 \mathrm{~h}$, cells were treated with lentiviral particles expressing shNONtg or shARID1A at a multiplicity of infection of 100 . Media was replaced after $24 \mathrm{~h}$. At $48 \mathrm{~h}$ post transfection, cells were trypsinized, and $100 \mu \mathrm{L}$ of cell mixture containing 30,000 cells and $0.3 \mathrm{mg} / \mathrm{mL}$ Matrigel was seeded into transwell plates $(8 \mu \mathrm{m}$ pore polycarbonate membrane, Corning) pre-coated with $100 \mu \mathrm{L}$ of $0.3 \mathrm{mg} / \mathrm{mL}$ Matrigel. After $1 \mathrm{~h}$, serum-free DMEM/F12 1\% P/S, $1 \%$ L-glutamine media was added to the top chamber and DMEM/F12, 5\% FBS, 1\% P/S, $1 \%$ L-glutamine was added to the bottom chamber. After $16 \mathrm{~h}$, transwell units were transferred to plates containing $4 \mu \mathrm{g} / \mathrm{mL}$ calcein AM in DMEM/F12. After $1 \mathrm{~h}$, media was aspirated from the top chamber and unmigrated cells were removed with a cotton swab. Images were collected using a Nikon Eclipse Ti microscope in five non-overlapping fields per well. ImageJ software (National Institutes of Health) was used to quantify cells based on size and intensity.

Western blotting. Protein lysates were quantified using the Micro BCA Protein Assay Kit (ThermoFisher) and a FlexSystem 3 plate reader. Protein lysates were run on a $4-15 \%$ gradient sodium dodecyl sulfate polyacrylamide gel electrophoresis (SDS-PAGE) gel (BioRad) and transferred to PVDF membrane using the TransBlot Turbo system (BioRad). Primary antibodies were used at the following dilutions: 1:1000 ARID1A (D2A8U) (12354, Cell Signaling); 1:1000 Akt (4691, Cell Signaling); 1:1000 $\beta$-Actin (8457, Cell Signaling); E-Cadherin (3195, Cell Signaling); 1:2000 Phospho-Akt (Ser473) (4060, Cell Signaling); 1:1000 Slug (9585, Cell Signaling); 1:1000 Snail (3879, Cell Signaling); 1:1000 Twist1 (T6451, Sigma); 1:100 ARID1B (sc-32762, Santa Cruz); 1:1000 Brg1 (ab110641, Abcam); 1:1000 BRM (11966, Cell Signaling); 1:100 ARID1A (PSG3) (sc-32761, Santa Cruz). Horseradish peroxidase (HRP) conjugated secondary antibodies (Cell Signaling) were used at a dilution of 1:2000. Clarity Western ECL Substrate (BioRad) was used for protein band visualization, and western blot exposures were captured using the ChemiDoc XRS + imaging system (BioRad). Uncropped western blot images can be found in Supplementary Fig. 7.

Chromatin immunoprecipitation. Wild-type $12 \mathrm{Z}$ cells were treated with $1 \%$ formaldehyde in DMEM/F12 media for 10 min at room temperature. Formaldehyde was quenched by the addition of $0.125 \mathrm{M}$ Glycine and incubation for $5 \mathrm{~min}$ at room temperature, followed by wash with PBS. In all, $1 \times 10^{7}$ crosslinked cells were used per IP. Chromatin from crosslinked cells was fractionated by digestion with micrococcal nuclease using the SimpleChIP Enzymatic Chromatin IP Kit (Cell Signaling) as per the manufacturers' instructions, followed by $30 \mathrm{~s}$ of sonication. IPs were performed using the SimpleChIP Enzymatic Chromatin IP Kit per the manufacturers' instructions with 1:100 anti-ARID1A (D2A8U) (12354, Cell Signaling). Crosslinks were reversed with $0.4 \mathrm{mg} / \mathrm{mL}$ Proteinase $\mathrm{K}$ (ThermoFisher) and $0.2 \mathrm{M} \mathrm{NaCl}$ at $65^{\circ} \mathrm{C}$ for $2 \mathrm{~h}$. DNA was purified using the ChIP DNA Clean \& Concentrator Kit (Zymo).

Chromatin immunoprecipitation sequencing (ChIP-seq). Libraries for input and IP samples were prepared by the Van Andel Genomics Core from $10 \mathrm{ng}$ of input material and IP material using the KAPA Hyper Prep Kit (v5.16) (Kapa Biosystems, Wilmington, MA USA). Prior to PCR amplification, end repaired and Atailed DNA fragments were ligated to Bioo Scientific NEXTflex Adapters (Bioo Scientific, Austin, TX, USA). Quality and quantity of the finished libraries were assessed using a combination of Agilent DNA High Sensitivity chip (Agilent Technologies, Inc.), QuantiFluor dsDNA System (Promega Corp., Madison, WI, USA), and Kapa Illumina Library Quantification qPCR assays (Kapa Biosystems). Individually indexed libraries were pooled and $75 \mathrm{bp}$, single-end sequencing was performed on an Illumina NextSeq 500 sequencer using 75 cycle HO sequencing kits (v2) (Illumina Inc., San Diego, CA, USA), with all libraries run across two flow cells to return a minimum read depth of $80 \mathrm{M}$ reads per input library and $40 \mathrm{M}$ read per IP library. Base calling was done by Illumina NextSeq Control Software (NCS) v2.0 and output of NCS was demultiplexed and converted to FastQ format with Illumina Bcl2fastq v1.9.0.

ChIP-seq analysis. Technical replicate libraries were combined across flow cells and trimmed with cutadapt and Trim Galore! followed by quality control analysis via FastQC. Trimmed reads were aligned to GRCh38.p12 reference genome via Bowtie $2^{74}$ with flag '-very-sensitive'. Reads were then sorted and indexed with samtools ${ }^{76}$. PCR duplicates were removed with Picard MarkDuplicates (http:// broadinstitute.github.io/picard/), and again sorted and indexed. MACS2 ${ }^{80}$ was used to call broad peaks with FDR $<0.05$ threshold on each ChIP replicate against the input control, and the resulting peaks were repeat-masked by blacklist filtering ${ }^{81}$. A naive overlap peak set, as defined by ENCODE, was constructed by combining replicates and calling broad peaks on pooled BAM files followed by intersectBed to select for peaks of at least $50 \%$ overlap with each biological replicate. Naive overlapping ChIP peaks were annotated by HOMER, and de novo motif enrichment and genome ontology were performed on genome-wide and promoter (within $3 \mathrm{~kb}$ of a TSS) peak sets. Overlapping genes between ChIP/ATAC and ChIP/ATAC/RNA were selected by the presence of a significant ChIP peak and differentially accessible promoter ATAC peak $(\mathrm{FDR}<0.05)$ located in the same promoter region (within $3 \mathrm{~kb}$ of TSS).

Co-immunoprecipitation (co-IP). Small-scale nuclear extracts and co-IPs from wild-type $12 \mathrm{Z}$ cells were performed ${ }^{20}$. Briefly, Protein A or Protein G Dynabeads (Invitrogen) were conjugated with anti-ARID1A (D2A8U) (12354, Cell Signaling) anti-ARID1A (PSG3) (sc-32761, Santa Cruz), or anti-ARID1B (E9J4T) (92964, Cell Signaling) in PBS $+0.5 \%$ BSA overnight at $4 \mathrm{C}$. Four-hundred micrograms of nuclear lysate was added to a final volume of $1 \mathrm{~mL}$ IP buffer $(20 \mathrm{mM}$ HEPES [pH 7.9], $250 \mathrm{mM} \mathrm{KCl}, 10 \%$ glycerol, $0.2 \mathrm{mM}$ EDTA, $0.1 \%$ Tween-20, $0.5 \mathrm{mM}$ DTT, $0.5 \mathrm{mM}$ PMSF), clarified by high-speed centrifugation and added to antibodyconjugated beads (D2A8U, 1:200; PSG3, 1:40; E9J4T, 1:200) and incubated 
overnight at $4{ }^{\circ} \mathrm{C}$. IP samples were washed in a series of IP buffers with varying salt concentrations as follows: $150 \mathrm{mM} \mathrm{KCl}, 300 \mathrm{mM} \mathrm{KCl}, 500 \mathrm{mM} \mathrm{KCl}, 300 \mathrm{mM}$ $\mathrm{KCl}, 100 \mathrm{mM} \mathrm{KCl}$. IP samples were washed a final time in $60 \mathrm{mM} \mathrm{KCl}$ IP buffer in the absence of EDTA or Tween-20. Proteins were eluted twice with $100 \mathrm{mM}$ glycine $\mathrm{pH} 2.5$ on ice and neutralized by the addition of 1:10 (v:v) of $1 \mathrm{M}$ Tris- $\mathrm{HCl}$ $\mathrm{pH} 8.0$.

Co-IP followed by mass spectrometry. Nuclear lysates from wild-type $12 \mathrm{Z}$ cells were prepared as described in the previous section. Protein A Dynabeads (Invitrogen) were conjugated with $8.3 \mu \mathrm{g}$ anti-ARID1A (D2A8U) (12354, Cell Signaling) or IgG $(2729$, Cell Signaling) in PBS $+0.5 \%$ BSA $+0.01 \%$ Tween- 20 overnight at $4{ }^{\circ} \mathrm{C}$. Antibody-bead conjugates were crosslinked in $\mathrm{BS}^{3}$ (ThermoFisher) as described by the manufacturer protocol, and excess unlinked antibody was removed by one wash of $0.11 \mathrm{M}$ glycine followed by quenching with Tris-HCl. $4.3 \mathrm{mg}$ of nuclear lysate was added to a final volume of $14 \mathrm{~mL}$ IP buffer $(20 \mathrm{mM}$ HEPES [pH 7.9], $150 \mathrm{mM} \mathrm{KCl,} \mathrm{10 \%} \mathrm{glycerol,} 0.2 \mathrm{mM}$ EDTA, $0.1 \%$ Tween-20, $0.5 \mathrm{mM}$ DTT, $0.5 \mathrm{mM}$ PMSF) and clarified by high-speed centrifugation. Diluted nuclear lysate was added to antibody-crosslinked beads and incubated overnight at $4{ }^{\circ} \mathrm{C}$. IP samples were washed in an IP buffer series with varying salt concentrations as follows: twice with $150 \mathrm{mM} \mathrm{KCl}$, three times with $300 \mathrm{mM} \mathrm{KCl}$, twice with $100 \mathrm{mM} \mathrm{KCl}$. IP samples were washed a final time in $60 \mathrm{mM} \mathrm{KCl}$ IP buffer in the absence of EDTA or Tween-20. Proteins were eluted in 2x Laemmli + $100 \mu \mathrm{M}$ DTT at $70{ }^{\circ} \mathrm{C}$ for $10 \mathrm{~min}$. Eluates were processed for short-gel SDS-PAGE and mass spectrometry by the University of Massachusetts Mass Spectrometry core.

Mass spectrometry analysis. All MS/MS samples were analyzed using Mascot (version 2.1.1.21, Matrix Science, London, UK). Mascot was set-up to search UniProtKB Swiss-Prot (Human) assuming the digestion enzyme as strict trypsin. Mascot was searched with a fragment ion mass tolerance of $0.050 \mathrm{Da}$ and a parent ion tolerance of 10.0 PPM. Carbamidomethyl of cysteine was specified in Mascot as a fixed modification. Gln- > pyro-Glu of glutamine and the N-terminus, oxidation of methionine and acetyl of the $\mathrm{N}$-terminus were specified in Mascot as variable modifications. Scaffold (version 4.8.8, Proteome Software Inc., Portland, OR) was used to validate MS/MS-based peptide and protein identifications. Peptide identifications were accepted if they could be established at $>85.0 \%$ probability by the Peptide Prophet algorithm ${ }^{102}$ with Scaffold delta-mass correction. Protein identifications were accepted if they could be established at greater than $99.0 \%$ probability and contained at least two identified peptides. Protein probabilities were assigned by the Protein Prophet algorithm ${ }^{103}$. Proteins that contained similar peptides and could not be differentiated based on MS/MS analysis alone were grouped to satisfy the principles of parsimony. Proteins sharing significant peptide evidence were grouped into clusters.

Reporting summary. Further information on research design is available in the Nature Research Reporting Summary linked to this article.

\section{Data availability}

The RNA-seq, ChIP-seq and ATAC-seq data have been deposited in the GEO database under the accession code GSE121198. All the other data supporting the findings of this study are available within the article and its supplementary information files and from the corresponding author upon reasonable request. Uncropped western blot images can be found in Supplementary Fig. 7. A reporting summary for this article is available as a Supplementary Information file.

Received: 12 December 2018 Accepted: 3 July 2019

Published online: 07 August 2019

\section{References}

1. Mihm, M., Gangooly, S. \& Muttukrishna, S. The normal menstrual cycle in women. Anim. Reprod. Sci. 124, 229-236 (2011).

2. Sorosky, J. I. Endometrial cancer. Obstet. Gynecol. 120, 383-397 (2012).

3. Chui, M. H., Wang, T. L. \& Shih, I. M. Endometriosis: benign, malignant, or something in between? Oncotarget 8, 78263-78264 (2017).

4. Abbott, J. A. Adenomyosis and abnormal uterine bleeding (AUB-A)pathogenesis, diagnosis, and management. Best Pr. Res Clin. Obstet. Gynaecol. 40, 68-81 (2017).

5. Kurman, R. J. \& Shih, IeM. The dualistic model of ovarian carcinogenesis: revisited, revised, and expanded. Am. J. Pathol. 186, 733-747 (2016).

6. Siegel, R. L., Miller, K. D. \& Jemal, A. Cancer statistics, 2018. CA Cancer J. Clin. 68, 7-30 (2018)

7. Renehan, A. G., Tyson, M., Egger, M., Heller, R. F. \& Zwahlen, M. Body-mass index and incidence of cancer: a systematic review and meta-analysis of prospective observational studies. Lancet 371, 569-578 (2008).
8. Wang, X., Haswell, J. R. \& Roberts, C. W. Molecular pathways: SWI/SNF (BAF) complexes are frequently mutated in cancer-mechanisms and potential therapeutic insights. Clin. Cancer Res 20, 21-27 (2014).

9. Kadoch, C. et al. Proteomic and bioinformatic analysis of mammalian SWI/ SNF complexes identifies extensive roles in human malignancy. Nat. Genet $\mathbf{4 5}$, 592-601 (2013).

10. Jones, S. et al. Frequent mutations of chromatin remodeling gene ARID1A in ovarian clear cell carcinoma. Science 330, 228-231 (2010).

11. Wiegand, K. C. et al. ARID1A mutations in endometriosis-associated ovarian carcinomas. N. Engl. J. Med 363, 1532-1543 (2010).

12. Guan, B. et al. Mutation and loss of expression of ARID1A in uterine lowgrade endometrioid carcinoma. Am. J. Surg. Pathol. 35, 625-632 (2011).

13. Wiegand, K. C. et al. Loss of BAF250a (ARID1A) is frequent in high-grade endometrial carcinomas. J. Pathol. 224, 328-333 (2011).

14. Mao, T. L. et al. Loss of ARID1A expression correlates with stages of tumor progression in uterine endometrioid carcinoma. Am. J. Surg. Pathol. 37, 1342-1348 (2013).

15. Yen, T. T. et al. Loss of ARID1A expression in endometrial samplings is associated with the risk of endometrial carcinoma. Gynecol. Oncol. 150, 426-431 (2018).

16. Anglesio, M. S. et al. Cancer-associated mutations in endometriosis without cancer. N. Engl. J. Med. 376, 1835-1848 (2017).

17. Suda, K. et al. Clonal expansion and diversification of cancer-associated mutations in endometriosis and normal endometrium. Cell Rep. 24, 1777-1789 (2018)

18. Gao, X. et al. ES cell pluripotency and germ-layer formation require the SWI/ SNF chromatin remodeling component BAF250a. Proc. Natl Acad. Sci. USA 105, 6656-6661 (2008).

19. Donehower, L. A. The p53-deficient mouse: a model for basic and applied cancer studies. Semin. Cancer Biol. 7, 269-278 (1996).

20. Chandler, R. L. et al. ARIDla-DNA interactions are required for promoter occupancy by SWI/SNF. Mol. Cell Biol. 33, 265-280 (2013).

21. Naumann, R. W. The role of the phosphatidylinositol 3-kinase (PI3K) pathway in the development and treatment of uterine cancer. Gynecol. Oncol. 123, 411-420 (2011).

22. Cancer Genome Atlas Research, N. et al. Integrated genomic characterization of endometrial carcinoma. Nature 497, 67-73 (2013).

23. Takeda, T. et al. ARID1A gene mutation in ovarian and endometrial cancers (Review). Oncol. Rep. 35, 607-613 (2016).

24. Berg, A. et al. Molecular profiling of endometrial carcinoma precursor, primary and metastatic lesions suggests different targets for treatment in obese compared to non-obese patients. Oncotarget 6, 1327-1339 (2015).

25. Perets, R. et al. Transformation of the fallopian tube secretory epithelium leads to high-grade serous ovarian cancer in Brca;Tp53;Pten models. Cancer Cell 24, 751-765 (2013)

26. $\mathrm{Wu}, \mathrm{R}$. et al. Impact of oviductal versus ovarian epithelial cell of origin on ovarian endometrioid carcinoma phenotype in the mouse. J. Pathol. 240, 341-351 (2016).

27. Zhai, Y. et al. High-grade serous carcinomas arise in the mouse oviduct via defects linked to the human disease. J. Pathol. 243, 16-25 (2017).

28. Zhang, R., Fukumoto, T. \& Magno, E. SWI/SNF complexes in ovarian cancer: mechanistic insights and therapeutic implications. Mol. Cancer Res 16, 1819-1825 (2018).

29. Guan, B. et al. Roles of deletion of Aridla, a tumor suppressor, in mouse ovarian tumorigenesis. J. Natl. Cancer Inst. 106, dju146 (2014).

30. Chandler, R. L. et al. Coexistent ARID1A-PIK3CA mutations promote ovarian clear-cell tumorigenesis through pro-tumorigenic inflammatory cytokine signalling. Nat. Commun. 6, 6118 (2015).

31. Wu, J. N. \& Roberts, C. W. ARID1A mutations in cancer: another epigenetic tumor suppressor? Cancer Disco. 3, 35-43 (2013).

32. Daikoku, T. et al. Lactoferrin-iCre: a new mouse line to study uterine epithelial gene function. Endocrinology 155, 2718-2724 (2014).

33. Adams, J. R. et al. Cooperation between Pik3ca and p53 mutations in mouse mammary tumor formation. Cancer Res. 71, 2706-2717 (2011).

34. Joshi, A., Miller, C. Jr., Baker, S. J. \& Ellenson, L. H. Activated mutant pl10alpha causes endometrial carcinoma in the setting of biallelic Pten deletion. Am. J. Pathol. 185, 1104-1113 (2015).

35. Zhang, Y. et al. Prognostic role of hormone receptors in endometrial cancer: systematic review and meta-analysis. World J. Surg. Oncol. 13, 208 (2015).

36. Kyo, S. et al. High twist expression is involved in infiltrative endometrial cancer and affects patient survival. Hum. Pathol. 37, 431-438 (2006).

37. Mak, M. P. et al. A patient-derived, pan-cancer EMT signature identifies global molecular alterations and immune target enrichment following epithelial-to-mesenchymal transition. Clin. Cancer Res. 22, 609-620 (2016).

38. Nieto, M. A., Huang, R. Y., Jackson, R. A. \& Thiery, J. P. Emt: 2016. Cell 166, 21-45 (2016).

39. Buenrostro, J. D., Giresi, P. G., Zaba, L. C., Chang, H. Y. \& Greenleaf, W. J. Transposition of native chromatin for fast and sensitive epigenomic profiling 
of open chromatin, DNA-binding proteins and nucleosome position. Nat. Methods 10, 1213-1218 (2013).

40. Zeitvogel, A., Baumann, R. \& Starzinski-Powitz, A. Identification of an invasive, $\mathrm{N}$-cadherin-expressing epithelial cell type in endometriosis using a new cell culture model. Am. J. Pathol. 159, 1839-1852 (2001).

41. Kelso, T. W. R. et al. Chromatin accessibility underlies synthetic lethality of SWI/SNF subunits in ARID1A-mutant cancers. Elife 6, e30506 (2017).

42. Vierbuchen, T. et al. AP-1 transcription factors and the BAF complex mediate signal-dependent enhancer selection. Mol. Cell 68, 1067-1082 e12 (2017).

43. Kim, M., Lu, F. \& Zhang, Y. Loss of HDAC-mediated repression and gain of NF-kappaB activation underlie cytokine induction in ARID1A- and PIK3CAmutation-driven ovarian cancer. Cell Rep. 17, 275-288 (2016).

44. Mirantes, C. et al. Epithelial-to-mesenchymal transition and stem cells in endometrial cancer. Hum. Pathol. 44, 1973-1981 (2013).

45. Friedl, P. \& Gilmour, D. Collective cell migration in morphogenesis, regeneration and cancer. Nat. Rev. Mol. Cell Biol. 10, 445-457 (2009).

46. Mamo, A. et al. An integrated genomic approach identifies ARID1A as a candidate tumor-suppressor gene in breast cancer. Oncogene 31, 2090-2100 (2012).

47. Zang, Z. J. et al. Exome sequencing of gastric adenocarcinoma identifies recurrent somatic mutations in cell adhesion and chromatin remodeling genes. Nat. Genet. 44, 570-574 (2012).

48. Guichard, C. et al. Integrated analysis of somatic mutations and focal copynumber changes identifies key genes and pathways in hepatocellular carcinoma. Nat. Genet. 44, 694-698 (2012).

49. Sun, X. et al. Aridla has context-dependent oncogenic and tumor suppressor functions in liver cancer. Cancer Cell 32, 574-589 e6 (2017).

50. Raab, J. R., Resnick, S. \& Magnuson, T. Genome-wide transcriptional regulation mediated by biochemically distinct SWI/SNF complexes. PLoS Genet. 11, e1005748 (2015).

51. Rafati, H. et al. Repressive LTR nucleosome positioning by the BAF complex is required for HIV latency. PLoS Biol. 9, e1001206 (2011).

52. Desouki, M. M. et al. Differential vimentin expression in ovarian and uterine corpus endometrioid adenocarcinomas: diagnostic utility in distinguishing double primaries from metastatic tumors. Int. J. Gynecol. Pathol. 33, 274-281 (2014).

53. Menkhorst, E. et al. Galectin-7 is elevated in endometrioid (type I) endometrial cancer and promotes cell migration. Oncol. Lett. 16, 4721-4728 (2018).

54. Rutherford, E. J., Hill, A. D. K. \& Hopkins, A. M. Adhesion in physiological, benign and malignant proliferative states of the endometrium: microenvironment and the clinical big picture. Cells 7, E43 (2018).

55. Euscher, E. et al. The pattern of myometrial invasion as a predictor of lymph node metastasis or extrauterine disease in low-grade endometrial carcinoma. Am. J. Surg. Pathol. 37, 1728-1736 (2013).

56. Morice, P., Leary, A., Creutzberg, C., Abu-Rustum, N. \& Darai, E. Endometrial cancer. Lancet 387, 1094-1108 (2016).

57. Ismiil, N. D. et al. Adenomyosis is associated with myometrial invasion by FIGO 1 endometrial adenocarcinoma. Int J. Gynecol. Pathol. 26, 278-283 (2007).

58. Polyak, K. \& Weinberg, R. A. Transitions between epithelial and mesenchymal states: acquisition of malignant and stem cell traits. Nat. Rev. Cancer $\mathbf{9}$, 265-273 (2009).

59. Zeisberg, M. \& Neilson, E. G. Biomarkers for epithelial-mesenchymal transitions. J. Clin. Invest 119, 1429-1437 (2009).

60. Li, C. et al. ARID1A gene knockdown promotes neuroblastoma migration and invasion. Neoplasma 64, 367-376 (2017).

61. Yan, H. B. et al. Reduced expression of the chromatin remodeling gene ARID1A enhances gastric cancer cell migration and invasion via downregulation of E-cadherin transcription. Carcinogenesis 35, 867-876 (2014).

62. Lakshminarasimhan, R. et al. Down-regulation of ARID1A is sufficient to initiate neoplastic transformation along with epigenetic reprogramming in non-tumorigenic endometriotic cells. Cancer Lett. 401, 11-19 (2017).

63. Lambert, A. W., Pattabiraman, D. R. \& Weinberg, R. A. Emerging biological principles of metastasis. Cell 168, 670-691 (2017).

64. Bartley, J., Julicher, A., Hotz, B., Mechsner, S. \& Hotz, H. Epithelial to mesenchymal transition (EMT) seems to be regulated differently in endometriosis and the endometrium. Arch. Gynecol. Obstet. 289, 871-881 (2014).

65. Martin, M. Cutadapt removes adapter sequences from high-throughput sequencing reads. EMBnet. J. 17, 10-12 (2011).

66. Andrews, S. FastQC: A Quality Control Tool for High Throughput Sequence Data. http://www.bioinformatics.babraham.ac.uk/projects/fastqc (2010).

67. Harrow, J. et al. GENCODE: the reference human genome annotation for The ENCODE Project. Genome Res. 22, 1760-1774 (2012).

68. Dobin, A. et al. STAR: ultrafast universal RNA-seq aligner. Bioinformatics 29, 15-21 (2013).
69. Love, M. I., Huber, W. \& Anders, S. Moderated estimation of fold change and dispersion for RNA-seq data with DESeq2. Genome Biol. 15, 550 (2014).

70. Love, M. I., Anders, S., Kim, V. \& Huber, W. RNA-Seq workflow: genelevel exploratory analysis and differential expression. F1000Res 4, 1070 (2015).

71. Ignatiadis, N., Klaus, B., Zaugg, J. B. \& Huber, W. Data-driven hypothesis weighting increases detection power in genome-scale multiple testing. Nat. Methods 13, 577-580 (2016).

72. Buenrostro, J. D., Wu, B., Chang, H. Y. \& Greenleaf, W. J. ATAC-seq: method for assaying chromatin accessibility genome-wide. Curr. Protoc. Mol. Biol. 109, 1-9 (2015). 2129.

73. Ackermann, A. M., Wang, Z., Schug, J., Naji, A. \& Kaestner, K. H. Integration of ATAC-seq and RNA-seq identifies human alpha cell and beta cell signature genes. Mol. Metab. 5, 233-244 (2016).

74. Langmead, B. \& Salzberg, S. L. Fast gapped-read alignment with Bowtie 2. Nat Methods 9, 357-359 (2012).

75. Lander, E. S. et al. Initial sequencing and analysis of the human genome. Nature 409, 860-921 (2001).

76. Li, H. et al. The sequence alignment/map format and SAMtools. Bioinformatics 25, 2078-2079 (2009).

77. Ou, J. et al. ATACseqQC: a Bioconductor package for post-alignment quality assessment of ATAC-seq data. BMC Genom. 19, 169 (2018).

78. Daley, T. \& Smith, A. D. Predicting the molecular complexity of sequencing libraries. Nat. Methods 10, 325-327 (2013).

79. Quinlan, A. R. \& Hall, I. M. BEDTools: a flexible suite of utilities for comparing genomic features. Bioinformatics 26, 841-842 (2010).

80. Zhang, Y. et al. Model-based analysis of ChIP-Seq (MACS). Genome Biol. 9 , R137 (2008)

81. Consortium, E. P. An integrated encyclopedia of DNA elements in the human genome. Nature 489, 57-74 (2012).

82. Lun, A. T. \& Smyth, G. K. csaw: a Bioconductor package for differential binding analysis of ChIP-seq data using sliding windows. Nucl. Acids Res. 44, e45 (2016).

83. Robinson, M. D., McCarthy, D. J. \& Smyth, G. K. edgeR: a Bioconductor package for differential expression analysis of digital gene expression data. Bioinformatics 26, 139-140 (2010).

84. Heinz, S. et al. Simple combinations of lineage-determining transcription factors prime cis-regulatory elements required for macrophage and B cell identities. Mol. Cell 38, 576-589 (2010).

85. Gao, J. et al. Integrative analysis of complex cancer genomics and clinical profiles using the cBioPortal. Sci. Signal 6, pl1 (2013).

86. Li, B. \& Dewey, C. N. RSEM: accurate transcript quantification from RNA-Seq data with or without a reference genome. BMC Bioinforma. 12, 323 (2011).

87. Ritchie, M. E. et al. limma powers differential expression analyses for RNA sequencing and microarray studies. Nucl. Acids Res. 43, e47 (2015).

88. Grossman, R. L. et al. Toward a shared vision for cancer genomic data. $N$. Engl. J. Med 375, 1109-1112 (2016).

89. Subramanian, A. et al. Gene set enrichment analysis: a knowledge-based approach for interpreting genome-wide expression profiles. Proc. Natl Acad. Sci. USA 102, 15545-15550 (2005).

90. Liberzon, A. et al. The Molecular Signatures Database (MSigDB) hallmark gene set collection. Cell Syst. 1, 417-425 (2015).

91. Barbie, D. A. et al. Systematic RNA interference reveals that oncogenic KRASdriven cancers require TBK1. Nature 462, 108-112 (2009).

92. Yu, G., Wang, L. G., Han, Y. \& He, Q. Y. clusterProfiler: an R package for comparing biological themes among gene clusters. OMICS 16, 284-287 (2012).

93. Maglott, D., Ostell, J., Pruitt, K. D. \& Tatusova, T. Entrez Gene: gene-centered information at NCBI. Nucl. Acids Res. 33, D54-D58 (2005)

94. Durinck, S., Spellman, P. T., Birney, E. \& Huber, W. Mapping identifiers for the integration of genomic datasets with the R/Bioconductor package biomaRt. Nat. Protoc. 4, 1184-1191 (2009).

95. Durinck, S. et al. BioMart and Bioconductor: a powerful link between biological databases and microarray data analysis. Bioinformatics 21, 3439-3440 (2005)

96. Wickham, H. Ggplot2: Elegant Graphics for Data Analysis, 1-212 (SpringerVerlag New York, 2009); https://doi.org/10.1007/978-0-387-98141-3.

97. Gu, Z., Eils, R. \& Schlesner, M. Complex heatmaps reveal patterns and correlations in multidimensional genomic data. Bioinformatics 32, 2847-2849 (2016).

98. Larsson, J. eulerr: Area-Proportional Euler and Venn Diagrams with Ellipses. $R$ package version 5.1.0. https://cran.r-project.org/package=eulerr (2019).

99. Team, R. C. R.: A Language and Environment for Statistical Computing. $R$ Foundation for Statistical Computing (Team, R. C., Vienna, Austria, 2018).

100. Team, B.C. \& Maintainer, B. P. TxDb.Hsapiens.UCSC.hg38.knownGene: Annotation package for $T x D b$ object(s). R package version 3.4.0. (Team, B.C. \& Maintainer, B. P., 2016) 
101. Zhao, J. J. et al. The oncogenic properties of mutant p110alpha and p110beta phosphatidylinositol 3-kinases in human mammary epithelial cells. Proc. Natl Acad. Sci. USA 102, 18443-18448 (2005).

102. Keller, A., Nesvizhskii, A. I., Kolker, E. \& Aebersold, R. Empirical statistical model to estimate the accuracy of peptide identifications made by MS/MS and database search. Anal. Chem. 74, 5383-5392 (2002).

103. Nesvizhskii, A. I., Keller, A., Kolker, E. \& Aebersold, R. A statistical model for identifying proteins by tandem mass spectrometry. Anal. Chem. $\mathbf{7 5}$, 4646-4658 (2003).

\section{Acknowledgements}

We thank Drs. Kathy Cho, Peter Laird, John Risinger, Jeff MacKeigan, Thomas McFall, Michael Anglesio, David Huntsman, and Ie-Ming Shih for helpful discussions. We thank the Van Andel Genomics Core for providing sequencing facilities and services. We thank the Van Andel Research Institute Histology and Pathology Core for the histology services, and Dr. Galen Hostetter for his assistance with mouse tumor pathology. We gratefully acknowledge the Mass Spectrometry Facility at the University of Massachusetts Medical School for assistance with the proteomic measurements. M.R.W. was supported by an American Cancer Society Postdoctoral Fellowship (PF-17-163-02-DDC). R.L.C was supported by an Innovative Translational Grant from the Mary Kay Foundation (026-16) and Liz Tilberis Early Career Award from the Ovarian Cancer Research Fund Alliance (OCRFA) (457446).

\section{Author contributions}

Conceptualization: M.R.W. and R.L.C.; Investigation: M.R.W., J.J.R., J.H. and G.E.W.; Methodology: M.R.W., J.J.R., M.A., B.J., A.L.P., H.S., J.M.T. and R.L.C.; Resources: M.R., J.K., R.S., N.R.J., and A.T.F.; Formal analysis: M.R.W. and J.J.R.; Data curation: J.J.R.; Writing-original draft: M.R.W., J.J.R., and R.L.C.; Writing-review and editing: M.R.W., J.J.R., J.H., G.E.W., B.J., A.L.P., H.S., R.E.L., J.M.T., A.T.F. and R.L.C.; Funding acquisition: M.R.W. and R.L.C.; Supervision: R.L.C.

\section{Additional information}

Supplementary Information accompanies this paper at https://doi.org/10.1038/s41467 019-11403-6.

Competing interests: The authors declare no competing interests.

Reprints and permission information is available online at http://npg.nature.com/ reprintsandpermissions/

Peer review information: Nature Communications thanks Eva Colas and other anonymous reviewer(s) for their contribution to the peer review of this work.

Publisher's note: Springer Nature remains neutral with regard to jurisdictional claims in published maps and institutional affiliations.

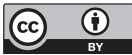

Open Access This article is licensed under a Creative Commons Attribution 4.0 International License, which permits use, sharing, adaptation, distribution and reproduction in any medium or format, as long as you give appropriate credit to the original author(s) and the source, provide a link to the Creative Commons license, and indicate if changes were made. The images or other third party material in this article are included in the article's Creative Commons license, unless indicated otherwise in a credit line to the material. If material is not included in the article's Creative Commons license and your intended use is not permitted by statutory regulation or exceeds the permitted use, you will need to obtain permission directly from the copyright holder. To view a copy of this license, visit http://creativecommons.org/ licenses/by/4.0/.

(C) The Author(s) 2019 